\title{
Kinetic Estimate of the Shielding of Resonant Magnetic Field Perturbations by the Plasma in DIII-D
}

\author{
Martin F. Heyn ${ }^{1}$, Ivan B. Ivanov ${ }^{1,2}$, Sergei V. Kasilov ${ }^{1,3}$, \\ Winfried Kernbichler ${ }^{1}$, Ilon Joseph ${ }^{4}$, Richard A. Moyer ${ }^{4}$, Alexey M. Runov ${ }^{5}$ \\ ${ }^{1}$ Association EURATOM-ÖAW, Institut für Theoretische \\ Physik - Computational Physics, Technische Universität Graz \\ Petersgasse 16, A-8010 Graz, Austria \\ ${ }^{2}$ Petersburg Nuclear Physics Institute \\ 188300, Gatchina, Leningrad Region, Russia \\ ${ }^{3}$ Institute of Plasma Physics \\ National Science Center "Kharkov Institute of Physics and Technology" \\ ul. Akademicheskaya 1, 61108 Kharkov, Ukraine \\ ${ }^{4}$ University of California - San Diego, La Jolla, CA, USA \\ ${ }^{5}$ Max Planck Institut für Plasmaphysik, EURATOM Association, \\ Teilinstitut Greifswald, Germany
}

\begin{abstract}
Effects of linear plasma response currents on non-axisymmetric magnetic field perturbations from the I-coil used for Edge Localized Mode mitigation in DIII-D tokamak are analyzed with the help of a kinetic plasma response model developed for cylindrical geometry. It is shown that these currents eliminate the ergodization of the magnetic field in the core plasma and reduce the size of the ergodic layer at the edge. A simple balance model is proposed which qualitatively reproduces the evolution of the plasma parameters in the pedestal region with the onset of the perturbation. It is suggested that the experimentally observed density pump-out effect in the long mean free path regime is the result of a combined action of ion orbit losses and magnetic field ergodization at the edge.

PACS numbers: 52.25.Dg, 52.25.Fi, 52.25.Gi, 52.25.Mq, 52.25.Xz, 52.35.Kt, 52.35.Py, 52.35.Qz, 52.55.Fa, 52.65.Ff, 52.65.Tt, 52.65.Vv
\end{abstract}




\section{INTRODUCTION}

A method of Edge Localized Mode (ELM) control using non-axisymmetric external magnetic field perturbations has been proposed and successfully tested in DIII-D ${ }^{1-6}$. Namely, some suppression of ELM's in high collisionality regimes has been reported in Refs. 2,3, suppression and full elimination of ELM's in low collisionality regimes has been reported in Refs. 4,5 and, in particular, for ITER similar plasma shape it has been reported in Ref. 6 .

An important issue using this method is to have a minimal influence of these perturbations on the transport in the core plasma. So far, theoretical studies of magnetic field ergodization $^{1,7,8}$ and heat transport ${ }^{1,8,9}$ in such ergodized fields in DIII-D used a vacuum perturbation field. Those calculations show that the magnetic field topology should be strongly affected by the perturbations ${ }^{1,7,8}$. In particular, splitting of heat and particle deposition profiles observed experimentally has been shown to be qualitatively consistent with magnetic footprints produced by splitting of the separatrix in the presence of non-axisymmetric perturbations ${ }^{7}$ and with the modelled 3D temperature distribution outside the separatrix ${ }^{8,9}$. At the same time, the modelling of heat transport using the Monte Carlo fluid code E3D ${ }^{10}$ performed in Refs. 8,9 shows a significant effect of the perturbations on plasma temperature both, in the pedestal region and deep in the core plasma. However, in the experiments the core plasma appears not to be affected by the perturbations. A possible explanation can be shielding of magnetic field perturbations by plasma response currents.

In this study this shielding effect, as well as the effect of the perturbation field on plasma rotation and other plasma parameter profiles is estimated with the help of the linear perturbation magnetic field model taking into account the plasma response currents in the kinetic approximation $^{11}$ which has been developed for cylindrical geometry. A particular experiment, DIII-D shot 126006, using the I-coil with even parity and current value $3.9 \mathrm{kA}$ is analyzed using the experimental equilibrium and plasma parameter profiles measured when the current in the $\mathrm{C}$-coil is set on. In parallel to calculations with the help of the kinetic model $^{11}$, a comparison is made with analytical results of linear drift-MHD model ${ }^{12}$. In addition, the applicability of linear and quasilinear approximations for the pertinent experiment is discussed.

The structure of the paper is as follows. In Section II coupling of the cylindrical model with the realistic equilibrium magnetic field computed with kinetic EFIT code ${ }^{13,14}$ and I- 
coil perturbation magnetic field provided by TRIP3D $\operatorname{code}^{1}$ is described, and results of the perturbation field penetration modelling and their comparison with the results of the reduced MHD model ${ }^{12}$ are presented. Results of the field line tracing in vacuum and in presence of plasma response currents are also presented in this section. In Section III quasilinear effects of the perturbation on plasma rotation and plasma parameter profiles are studied and the role of nonlinear effects is estimated. In Section IV the implications of the field line tracing results for the transport in the pedestal region are discussed. In Section $\mathrm{V}$ the results are summarized.

\section{PERTURBATION FIELD IN PRESENCE OF LINEAR PLASMA RESPONSE CURRENTS}

For modelling, the magnetic field is presented in the following form ${ }^{15}$

$$
\mathbf{B}=\mathbf{B}_{0}+\delta \mathbf{B}=\nabla \varphi \times \nabla \psi_{\text {pol }}+\nabla\left(\psi_{\text {tor }}+\tilde{A}_{\vartheta}\right) \times \nabla \vartheta
$$

where $\psi_{\text {tor }}$ and $\psi_{\text {pol }}$ are toroidal and poloidal magnetic fluxes (divided by $2 \pi$ ) of the unperturbed magnetic field $\mathbf{B}_{0}, \varphi$ is the azimuth (toroidal) angle of cylindrical coordinates $(R, \varphi, Z)$ and $\vartheta$ is the poloidal angle like variable of the flux coordinates $\left(\psi_{\text {tor }}, \vartheta, \varphi\right)$. Vector potential $\tilde{A}_{\vartheta}$ of the perturbation field $\delta \mathbf{B}$ is represented by a Fourier series in these flux variables,

$$
\tilde{A}_{\vartheta}=2 \operatorname{Re} \sum_{n=1}^{\infty} \sum_{m=-\infty}^{\infty} A_{\vartheta ; m, n}\left(\psi_{\text {tor }}\right) \mathrm{e}^{i m \vartheta+i n \varphi}
$$

Details of numerical computation of flux variables and complex Fourier amplitudes in the

vacuum case, $A_{\vartheta ; m, n}=A_{\vartheta ; m, n}^{(\mathrm{vac})}$, are presented in Appendices A and B. In presence of plasma response currents, Fourier amplitudes are expressed through the amplitudes in vacuum and the "form factors" $T_{m, n}$ as follows

$$
A_{\vartheta ; m, n}\left(\psi_{\text {tor }}\right)=A_{\vartheta ; m, n}^{(\mathrm{vac})}\left(\psi_{\text {tor }}\right) T_{m, n}\left(\psi_{\text {tor }}\right)
$$

\section{A. Estimations with help of a cylindrical model}

For estimation of form factors $T_{m, n}$, the model of an inhomogeneous periodic plasma cylinder with rotational transform of the unperturbed magnetic field, $B_{0}^{\vartheta}=B_{0}^{z} /\left(q R_{\text {axis }}\right)$, is introduced 
where the radial coordinate is related to the toroidal flux by $r=\sqrt{2 \psi_{\text {tor }} / B_{\text {axis }}}$, the $z$ coordinate is related to the toroidal angle by $z=\varphi R_{\text {axis }}$ and the azimuth $\vartheta$ is identified as poloidal angle. Here $B_{\text {axis }}=B_{0 \varphi} / R_{\text {axis }}$ is the unperturbed magnetic field value at the magnetic axis $R=R_{\text {axis }}$ (see also Appendix A). Profiles of the safety factor, $q(r)$, plasma density, $n_{e}(r)$, electron and ion temperature, $T_{e}(r)$ and $T_{i}(r)$, respectively, and of the toroidal rotation velocity, $V_{(i)}^{z}=R_{\text {axis }} V_{(i)}^{\varphi}$, correspond to DIII-D shot 126006. Given these profiles, the profile of the toroidal magnetic field, $B_{0}^{z}(r)$, is obtained from the equilibrium equation for the cylinder, the profile of the parallel plasma current density, $j_{0 \|}(r)$, follows from $q(r)$ and the profile of the equilibrium radial electric field is computed assuming zero poloidal plasma rotation velocity, $V_{(i)}^{\vartheta}=0$. All these profiles are smoothly extended outside the separatrix. The resulting profiles are shown in Figs. 1-3 as functions of radius $r$ and of the normalized toroidal flux $s=\psi_{\text {tor }} / \psi_{\text {tor }}^{(\mathrm{a})}=(r / a)^{2}$, where $\psi_{\text {tor }}^{(\mathrm{a})}$ is the toroidal flux value at the separatrix and $a$ is separatrix radius. It should be noted that directions of plasma current and toroidal magnetic field were opposite in the discharge 126006. Therefore, the coordinate system where $q>0$ used here is left-handed (resonant poloidal and toroidal wave numbers have opposite sign too). In order to avoid the ambiguity, directions of various velocities are explicitly related in the plots to the directions of parallel plasma current or poloidal magnetic field.

Outside the separatrix, plasma is assumed to be surrounded by the sheet current ("antenna") at $r=r_{A}$ and a perfectly conducting wall at $r=r_{W}>r_{A}$. Thus, ignoring poloidal coupling of Fourier modes, form factors are estimated with help of the cylindrical model as follows,

$$
T_{m, n}\left(\psi_{\text {tor }}\right)=\frac{B_{r}^{(\text {plas })}(r)}{B_{r}^{(v a c)}(r)},
$$

where $B_{r}^{(\text {plas })}$ and $B_{r}^{(v a c)}$ are the radial components of a single harmonic, $\propto \exp \left(i m \vartheta+i k_{z} z\right)$, of the perturbation magnetic field computed with plasma and in vacuum, respectively.

In order to show that radial positions of the antenna and of the wall do not influence $T_{m, n}$ if plasma pressure and current density are small in the region $r>r_{p}$ containing the antenna, $r_{p}<r_{A}$, the following ideal MHD equation for the "outer" solution of tearing mode theory ${ }^{16}$ is considered,

$$
\begin{aligned}
\frac{\mathrm{d}}{\mathrm{d} r} \frac{r}{k^{2}} \frac{\mathrm{d} B_{r}}{\mathrm{~d} r} & -r B_{r}\left(1-\frac{\mathrm{d}}{\mathrm{d} r} \frac{1}{r k^{2}}+F_{p}\right)=\frac{4 \pi r}{i c k_{\vartheta}} j_{z}^{(A)} \\
F_{p} & =\frac{r}{\mathbf{k} \cdot \mathbf{B}_{0}}\left[\frac{\mathrm{d}}{\mathrm{d} r} \frac{1}{r k^{2}} \frac{4 \pi}{c}\left(\mathbf{k} \times \mathbf{j}_{0}\right)_{r}+\frac{2}{r^{2} k^{2}}\left(k_{z} B_{0 z}^{\prime}+\frac{4 \pi k_{z}^{2}}{\mathbf{k} \cdot \mathbf{B}_{0}} \frac{\mathrm{d} p_{0}}{\mathrm{~d} r}\right)\right],
\end{aligned}
$$


where $\mathbf{k}=\mathbf{e}_{z} k_{z}+\mathbf{e}_{\vartheta} k_{\vartheta}, k_{z}=n / R, k_{\vartheta}=m / r, \mathbf{j}_{0}$ and $p_{0}$ are equilibrium plasma current and pressure and $j_{z}^{(A)}=I_{A} \delta\left(r-r_{A}\right)$ is z-component of the antenna current. Solutions for the homogeneous equation (5) regular at the cylinder axis in vacuum case, $F_{p}=0$, and with plasma are denoted with $u_{\text {int }}$ and $w$, respectively. Solution, which satisfies boundary condition at the wall in vacuum case is denoted with $u_{\text {ext }}$. In the region $r>r_{p}$ where $F_{p}$ can be ignored $w$ can be presented as $w=\alpha u_{\text {int }}+u_{\text {ext }}$ where $\alpha=$ Const. Solution of the inhomogeneous equation (5) in the region $r<r_{A}$ is

$$
B_{r}(r)=\frac{4 \pi i k^{2}\left(r_{A}\right) r_{A} I_{A} u_{\mathrm{ext}}\left(r_{A}\right) w(r)}{c m\left(w^{\prime}\left(r_{A}\right) u_{\mathrm{ext}}\left(r_{A}\right)-w\left(r_{A}\right) u_{\mathrm{ext}}^{\prime}\left(r_{A}\right)\right)} .
$$

Replacing here for the vacuum case $w$ and $w^{\prime}$ with $u_{\text {int }}$ and $u_{\text {int }}^{\prime}$, respectively, form factor (4) is obtained as

$$
T_{m, n}=\frac{w(r)}{\alpha u_{\mathrm{int}}(r)} .
$$

If a resonant magnetic surface, $q(r)=m / n$, is present in plasma, the ideal MHD equation (5) is not sufficient in the resonance layer. In this layer, a 4-th or higher order equation has to be solved which, in addition to the "fast mode" described by (5), describes also "slow", short scale, evanescent modes which correspond to shear Alfven and ion Bernstein modes. However additional solutions associated with slow modes and subject to the boundary conditions rapidly decrease with the distance from the resonance surface so that outside the resonance layer $w$ is the only important solution regular at the magnetic axis.

It should be noted that self-consistent equilibrium in cylindrical geometry leads to peculiarities of parallel current near the separatrix where $q$ formally diverges. A true $q$ profile is used only up to $q \approx 5.5$ and extended further outside assuming the parallel equilibrium current density to be finite and exponentially decaying outside the separatrix. The equilibrium results in a negative parallel current density $j_{0 \|}$ around the separatrix (see Fig. 2). This circumstance, however, does not have a significant influence on the results. If the equilibrium current density is fully ignored in the unperturbed distribution function, the resulting perturbation fields change by $50 \%$ or less which is significantly smaller than the effect of plasma response currents on those fields as shown below. Another circumstance which adds to the uncertainty at the edge is coupling of poloidal modes which becomes strong there but is fully omitted in straight cylinder or slab models. Therefore, the results should be considered as an estimate valid by order of magnitude. 


\section{B. Kinetic plasma response model}

The form factors (4) can be estimated by various plasma response models for the straight cylinder as well as slab models ${ }^{12,17-21}$. Here, the kinetic linear plasma response model of Ref. 11 is applied for this purpose. In this model, Maxwell equations,

$$
\nabla \times \tilde{\mathbf{E}}=\frac{i \omega}{c} \tilde{\mathbf{B}}, \quad \nabla \times \tilde{\mathbf{B}}=-\frac{i \omega}{c} \tilde{\mathbf{E}}+\frac{4 \pi}{c} \tilde{\mathbf{j}}
$$

are solved in cylindrical geometry retaining the Maxwell current and using a plasma response current $\tilde{\mathbf{j}}$ computed in the kinetic approximation with the help of a Hamiltonian formalism taking into account collisions by means of a Krook term. For a single spatial harmonic, $\tilde{E}_{i}, \tilde{j}^{i} \propto \exp \left(i m_{\vartheta} \vartheta+i k_{z} z\right)$, where the notation for the poloidal wave number is changed to $m_{\vartheta}$, the contra-variant components of the perturbed current density of a single particle species contributing to $\tilde{\mathbf{j}}$ in (9) is written through a differential conductivity operator as follows,

$$
\tilde{j}_{(N)}^{k}(r, \vartheta, z)=\frac{1}{r} \sum_{n, n^{\prime}=0}^{N}(-1)^{n} \frac{\partial^{n}}{\partial r^{n}}\left(r \sigma_{\left(n, n^{\prime}\right)}^{k l}(r, \mathbf{k}) \frac{\partial^{n^{\prime}}}{\partial r^{n^{\prime}}} \tilde{E}_{l}(r, \vartheta, z)\right),
$$

where $\tilde{E}_{l}$ are the co-variant components of the electric field,

$$
\begin{aligned}
\sigma_{\left(n, n^{\prime}\right)}^{k l}(r, \mathbf{k}) & =\frac{2 \pi i e^{2}}{r \omega} \sum_{m_{\phi}=-\infty}^{\infty} \int_{0}^{\infty \operatorname{sgn}(e)} \mathrm{d} J_{\perp} \int_{-\infty}^{\infty} \mathrm{d} u_{\|} \int_{0}^{\infty} \mathrm{d} r_{0} \delta\left(r-r_{g}\right) \frac{\partial\left(p_{\vartheta}, p_{z}\right)}{\partial\left(r_{0}, u_{\|}\right)}\left(a_{\alpha}^{k}(n)\right)_{\mathbf{m}}^{*}\left(a_{\beta}^{l}\left(n^{\prime}\right)\right)_{\mathbf{m}} \\
& \times \frac{1}{\mathbf{m} \cdot \Omega-\omega-i \nu} \Omega^{\alpha}\left((\omega-\mathbf{m} \cdot \boldsymbol{\Omega}) \frac{\partial f_{0}}{\partial J_{\beta}}+\Omega^{\beta} \mathbf{m} \cdot \frac{\partial f_{0}}{\partial \mathbf{J}}\right), \\
\left(a_{\alpha}^{k}(n)\right)_{\mathbf{m}} & =\frac{1}{2 \pi n !} \int_{-\pi}^{\pi} \mathrm{d} \phi \mathrm{e}^{-i m_{\phi} \phi}\left(\rho^{r}\right)^{n}\left(\frac{\partial x_{g}^{k}}{\partial \theta^{\alpha}} \sum_{l=0}^{N-n} \frac{1}{l !}(i \mathbf{k} \cdot \boldsymbol{\rho})^{l}+\frac{\partial \rho^{k}}{\partial \theta^{\alpha}} \sum_{l=0}^{N-n-1} \frac{1}{l !}(i \mathbf{k} \cdot \boldsymbol{\rho})^{l}\right),
\end{aligned}
$$

and where the second sum is zero if $N-n<1$. Here, the canonical angles and actions are $\boldsymbol{\theta}=(\phi, \vartheta, z)$ and $\mathbf{J}=\left(J_{\perp}, p_{\vartheta}, p_{z}\right)$ where $\phi$ is gyrophase, $J_{\perp} \approx m_{0} v_{\perp}^{2} /\left(2 \omega_{c}\right)$ is the perpendicular adiabatic invariant, $m_{0}$ is particle mass, $\omega_{c}$ is the cyclotron frequency, $\mathbf{m}=$ $\left(m_{\phi}, m_{\vartheta}, k_{z}\right)$ where $m_{\phi}$ is a cyclotron harmonic number, $\nu$ is the collision frequency and $\mathbf{k} \cdot \boldsymbol{\rho}=m_{\vartheta} \rho^{\vartheta}+k_{z} \rho^{z}$. The Larmor radius, $\boldsymbol{\rho}$, is defined expressing particle coordinates $x^{i}$ via canonical variables as $x^{i}=x_{g}^{i}+\rho^{i}$, where $x_{g}^{i}$ are guiding center coordinates independent of $\phi$ and the gyro-average of $\rho^{i}$ is zero. Integration variables $r_{0}$ and $u_{\|}$are defined through the co-variant components of the generalized momentum as

$$
p_{\vartheta, z}=p_{\vartheta, z}\left(r_{0}, u_{\|}\right)=\left(m_{0} \mathbf{h}\left(r_{0}\right) u_{\|}+\frac{e}{c} \mathbf{A}_{0}\left(r_{0}\right)+\frac{m_{0} c}{B_{0}\left(r_{0}\right)} \mathbf{h}\left(r_{0}\right) \times \nabla \Phi_{0}\left(r_{0}\right)\right)_{\vartheta, z},
$$


where $\mathbf{h}=\mathbf{B}_{0} / B_{0}$ and $\mathbf{B}_{0}=\nabla \times \mathbf{A}_{0}$, and canonical frequencies are $\boldsymbol{\Omega}=$ $\left(\omega_{c}, h^{\vartheta} u_{\|}+v_{E}^{\vartheta}, h^{z} u_{\|}+v_{E}^{z}\right)$ where $v_{E}^{i}$ are the contra-variant components of the electric drift velocity. The unperturbed distribution function is used in the form of an inhomogeneous drifting Maxwellian,

$$
f_{0}=\frac{n_{0}\left(r_{0}\right)}{\left(2 \pi m_{0} T_{0}\left(r_{0}\right)\right)^{3 / 2}} \exp \left(-\frac{\omega_{c}\left(r_{0}\right) J_{\perp}}{T_{0}\left(r_{0}\right)}-\frac{m_{0}\left(u_{\|}-V_{\|}\left(r_{0}\right)\right)^{2}}{2 T_{0}\left(r_{0}\right)}\right),
$$

where the parameters $n_{0}, T_{0}$ and $V_{\|}$for each species differ only by first order Larmor radius corrections from the equilibrium density, temperature, and parallel fluid velocity of the respective species. All these parameters and also the equilibrium electrostatic potential $\Phi_{0}$ are fully defined by the experimentally measured profiles shown in Fig. 1, the profile of the safety factor $q(r)$ and the reference magnetic field value $B_{\text {axis }}$.

The stiff set of Maxwell equations (9) is solved numerically using the re-orthonormalization procedure. Since even for a Larmor radius expansion of order $N=1$ used here for both, the electrons and the ions, explicit expression for the plasma response current is rather complex, the FORTRAN subroutines for the conductivity operator have been directly generated by the symbolic processor package MAPLE ${ }^{22}$. (As a check of this fully implicit approach, the results of the ideal MHD equation (5) are accurately reproduced by the code in the absence or away from the resonance surface.) The computations for the static perturbations in DIII-D are performed in a frame of reference moving along the Z-axis so that perturbation frequency $\omega$ is finite in this frame. In the non-relativistic limit, the magnetic field and the co-variant components of the force (torques) acting on the $\alpha$-species from the single harmonic of the perturbation field

$$
T_{(\alpha) \vartheta}^{(\mathrm{EM})}=\frac{m_{\vartheta}}{\omega} P_{\alpha}, \quad T_{(\alpha) \varphi}^{(\mathrm{EM})}=\frac{n}{\omega} P_{\alpha},
$$

are independent of the frame of reference. The absorbed power,

$$
P_{\alpha}=\frac{1}{2} \operatorname{Re} \int \mathrm{d}^{3} r \tilde{E}_{k}^{*} \tilde{j}_{(N)}^{k}
$$

where $\tilde{j}_{(N)}^{k}$ is the $\alpha$-species current density (10), scales linearly with $\omega$ when changing the reference frame.

As one can see, in contrast to the reduced MHD theory ${ }^{12,17,18,20}$, plasma neutrality and incompressibility are not enforced in the kinetic model, and the model should properly recover the compressible MHD results ${ }^{23}$. At the same time, any anomalous shear viscosity 
is not taken into account. Therefore, various regimes with large viscosity effects presented in Ref. 12 are missing in the present model.

The radial profiles of the form factor modules $\left|T_{m, n}\left(\psi_{\text {tor }}\right)\right|$ resulting from the kinetic model are shown in Fig. 4 for the I-coil main toroidal mode $n=3$. The resonant modes with $m_{\vartheta}=m \leq-4$ are reduced by more than one order of magnitude at corresponding rational flux surfaces, $m+n q\left(\psi_{\text {tor }}^{(\text {res })}\right)=0$. The non-resonant modes $-3 \leq m \leq-1$ show a similar trend as the resonant ones due to the properties of the ideal MHD response (in the absence of plasma rotation this response is described by Eq. (5)). In Fig. 5 the values of the form factor modules at the corresponding resonance surfaces $\left|T_{m, n}^{(\text {res })}\right|=\left|T_{m, n}\left(\psi_{\text {tor }}^{(\text {res })}\right)\right|$ are shown versus $s_{\text {res }}=\psi_{\text {tor }}^{(\text {res }} / \psi_{\text {tor }}^{(\text {a) }}$ and compared to the results of the drift-MHD theory of Ref. 12 . The latter results are given by Eq. (9) of Ref. 12 as $\left|T_{m, n}^{(\text {res })}\right|=|\Psi(t) / \Xi(t)|$ with substitution of local plasma parameters and wave numbers and putting the viscosity coefficient to $10^{4} \mathrm{~cm}^{2} \mathrm{~s}^{-1}$. The results agree for most of the resonant modes except for those in the regions with high perpendicular equilibrium electron fluid velocity where the mode frequency in the rest frame of the electrons exceeds the electron-ion collision frequency (see Fig. 6). One should note that the curve in Fig. 5 corresponding to drift MHD theory actually consists of few curves which correspond to relevant regimes of Ref. 12. Values of $\left|T_{m, n}^{(\text {res })}\right|$ for the "first semicollisional" regime which dominates here, as well as other observed regimes do not depend on the viscosity explicitly. It should also be noted that both models agree well in predicting the small screening of the magnetic field perturbations at the very edge of plasma due to the increasing shear and resistivity when approaching the separatrix.

\section{Relation between the electron and ion torque}

For further analysis, it is interesting to estimate how the torque from the perturbation is distributed between electrons and ions. Simple estimate can be obtained using the cold resistive MHD model where the linearized equation for the perturbed fluid velocity (ignoring the negligible unperturbed parallel velocity) is

$$
-i\left(\omega^{\prime}+i \nu_{\alpha}\right) \tilde{\mathbf{V}}_{\alpha}+\boldsymbol{\omega}_{c \alpha} \times \tilde{\mathbf{V}}_{\alpha}=\frac{e_{\alpha}}{m_{\alpha}} \tilde{\mathbf{E}}^{\prime}
$$

Here $\omega^{\prime}=\omega-\mathbf{k} \cdot \mathbf{V}_{E}=\omega-\omega_{E}$ is perturbation frequency in the frame of reference where the equilibrium electric field is zero (which is also the rest frame of $\alpha$ species), $\mathbf{V}_{E}$ is the electric 
drift velocity, $\boldsymbol{\omega}_{c \alpha}=e_{\alpha} \mathbf{B}_{0}\left(m_{\alpha} c\right)^{-1}$ is the cyclotron frequency, $e_{\alpha}$ and $m_{\alpha}$ are $\alpha$-species charge and mass, respectively and $\tilde{\mathbf{E}}^{\prime}$ is the electric field perturbation in the rest frame of $\alpha$ species. The collision frequency $\nu_{\alpha}$ is put for electrons to the electron-ion collision frequency, $\nu_{e}=\nu_{e i}$, and for ions it models the viscosity effect, $\nu_{i}=\mu_{\perp} \delta^{-2}$, where $\mu_{\perp}$ is the (generally anomalous) perpendicular viscosity coefficient and $\delta$ is the resonance layer width. Integrating over the volume work of the electric field on the response current, $\left(e_{\alpha} / 2\right) \operatorname{Re}\left[\tilde{\mathbf{E}}^{*} \cdot\left(n_{0 \alpha} \tilde{\mathbf{V}}_{\alpha}+\tilde{n}_{\alpha} \mathbf{V}_{E}\right)\right]$, one obtains for the total power absorbed by $\alpha$-species,

$$
P_{\alpha, \text { cold }}=\frac{\omega}{\omega^{\prime}} \frac{\omega_{p \alpha}^{2}}{8 \pi} \operatorname{Re} i \int \mathrm{d}^{3} r\left(\frac{\left|\tilde{E}_{\|}^{\prime}\right|^{2}}{\omega^{\prime}+i \nu_{\alpha}}+\frac{\left(\omega^{\prime}+i \nu_{\alpha}\right)\left|\tilde{\mathbf{E}}_{\perp}^{\prime}\right|^{2}}{\left(\omega^{\prime}+i \nu_{\alpha}\right)^{2}-\omega_{c \alpha}^{2}}+\frac{i \tilde{\mathbf{E}}_{\perp}^{*} \times \tilde{\mathbf{E}}_{\perp}^{\prime} \cdot \boldsymbol{\omega}_{c \alpha}}{\left(\omega^{\prime}+i \nu_{\alpha}\right)^{2}-\omega_{c \alpha}^{2}}\right)
$$

where $\omega_{p \alpha}=\left(4 \pi n_{0 \alpha} e_{\alpha}^{2} / m_{\alpha}\right)^{1 / 2}$ is plasma frequency of $\alpha$-species. The perturbation frequency in the laboratory frame $\omega$ is intendedly kept finite because it cancels in the expressions for the torque (14). When obtaining (17), the electric drift velocity $\mathbf{V}_{E}$ was assumed constant within the resonance layer where the main power absorption takes place. For electrons, only the largest first term (absorption due to the parallel conductivity) should be retained while for ions this term is always smaller than the corresponding term for electrons and should be ignored. The last (Hall) term should also be ignored for ions because it is small when compared to the second term. It can be seen that the resulting $P_{i, \text { cold }} \sim \int \mathrm{d}^{3} r n_{0 i} m_{i} \nu_{i} \tilde{V}_{i}^{2}$ is the work of the viscous force on the perturbed ion velocity $\tilde{V}_{i}=c\left|\tilde{E}_{\perp}^{\prime}\right| / B_{0}$. Thus, one obtains

$$
P_{e, \text { cold }} \sim S \delta \frac{\omega \omega_{p e}^{2} \nu_{e i}\left|\tilde{E}_{\|}^{\prime}\right|^{2}}{\omega^{\prime}\left(\omega^{\prime 2}+\nu_{e i}^{2}\right)}, \quad P_{i, \text { cold }} \sim S \delta \frac{\omega \omega_{p i}^{2} \nu_{i}\left|\tilde{\mathbf{E}}_{\perp}^{\prime}\right|^{2}}{\omega^{\prime} \omega_{c i}^{2}},
$$

where $S$ is the magnetic surface area. The electric field components can be estimated as components of the electrostatic field, $\left|\tilde{E}_{\|}^{\prime}\right| /\left|\tilde{\mathbf{E}}_{\perp}^{\prime}\right| \sim k_{\|} \delta$, expanding $k_{\|} \equiv \mathbf{k} \cdot \mathbf{h}$ around $r_{\text {res }}$ being the resonant surface radius, $k_{\|} \approx k_{\|}^{\prime} \delta$ where $k_{\|}^{\prime} \equiv \mathrm{d} k_{\|} / \mathrm{d} r=s_{B} k_{z} / r_{\text {res }}$ and $s_{B}=\left(r_{\text {res }} / q\right) \mathrm{d} q / \mathrm{d} r_{\text {res }}$ is the shear parameter. One can do this for the so called "constant psi" regimes which include most of the regimes in Ref. 12 except visco-inertial and inertial regimes. In the "constant psi" regimes, the inductive parallel electric field (the only one present at the resonant surface where $k_{\|}=0$ ) is approximately constant in the resonant layer and is balanced by the rapidly changing with radius parallel electrostatic field only outside the layer. Therefore, the total $\tilde{E}_{\|}^{\prime}$ can be estimated as the electrostatic field at distance $\delta$ from the resonant surface. It can be seen from Figs. 6 and 3 that $\omega_{e} \sim \omega_{E} \sim \omega_{i} \sim \nu_{e i}$. In this case one obtains from (14) 
and (18)

$$
\left(\frac{T_{(e) \varphi}^{(E M)}}{T_{(i) \varphi}^{(E M)}}\right)_{\text {cold }}=\frac{P_{e, \text { cold }}}{P_{i, \text { cold }}} \sim \frac{m_{i} \omega_{c i}^{2}\left|\tilde{E}_{\|}^{\prime}\right|^{2}}{m_{e} \nu_{e i} \nu_{i}\left|\tilde{\mathbf{E}}_{\perp}^{\prime}\right|^{2}} \sim\left(\frac{\delta}{\delta_{V R}}\right)^{6},
$$

where $\delta_{V R}=r_{\text {res }} \tau_{H}^{1 / 3} \tau_{R}^{-1 / 6} \tau_{V}^{-1 / 6}$ is the resonance layer width for the visco-resistive regime ${ }^{12,19}$,

$$
\tau_{H}=\frac{1}{s_{B} k_{z} v_{A}}, \quad \tau_{R}=\frac{4 \pi n_{0 e} e^{2} r_{\mathrm{res}}^{2}}{m_{e} \nu_{e i} c^{2}}, \quad \tau_{V}=\frac{r_{\mathrm{res}}^{2}}{\mu_{\perp}}
$$

are the Alfven, resistive and viscous times, respectively, and $v_{A}=B_{0}\left(4 \pi n_{0 i} m_{i}\right)^{-1 / 2}$ is the Alfven velocity. For finite electron temperatures, the electron pressure effect can be impor$\operatorname{tant}^{20}$ which limits the electron absorption region to $k_{\|} v_{T e}<\left(\omega_{E} \nu_{e i}\right)^{1 / 2}$ which has a width $\delta_{e} \sim\left(\omega_{E} \nu_{e i}\right)^{1 / 2}\left(v_{T e}\left|k_{\|}^{\prime}\right|\right)^{-1}$ where $v_{T e}=\left(T_{e} / m_{e}\right)^{1 / 2}$. Since $\omega_{E} \sim \nu_{e i}$, collisional electron damping is of the order of collisionless Landau damping which can be calculated from ${ }^{11}$

$$
\begin{aligned}
P_{e} & \approx \pi^{2} \frac{S m_{e}^{2} \omega}{r_{\text {res }}} \int_{0}^{\infty} \mathrm{d} r_{0} r_{0} \int_{-\infty}^{\infty} \mathrm{d} u_{\|} \int_{-\infty}^{0} \mathrm{~d} J_{\perp} \frac{\left|\omega_{c e}\right|}{T_{e}} f_{e 0} \delta\left(k_{\|} u_{\|}+\omega_{E}-\omega\right)\left|H_{\mathbf{m}}\right|^{2} \\
& \times\left[\omega-\omega_{e}+\frac{k_{\perp} T_{e}^{\prime}}{m_{e} \omega_{c e}}\left(\frac{5}{2}-\frac{\omega_{c e} J_{\perp}}{T_{e}}-\frac{m_{e} u_{\|}^{2}}{2 T_{e}}\right)\right]
\end{aligned}
$$

where $\left|H_{\mathbf{m}}\right| \approx\left|e_{e} u_{\|} \tilde{E}_{\|}^{\prime} / \omega_{E}\right|, \omega_{e}=\omega_{E}+\omega_{* e}$ is stationary perturbation frequency in the electron rest frame and $T_{e}^{\prime} \equiv \mathrm{d} T_{e} / \mathrm{d} r_{0}$. The parallel fluid velocity $V_{\|}$in expression (13) for the equilibrium distribution function $f_{e 0}$ has negligible effect and, therefore, is ignored there. Assuming that $\tilde{E}_{\|}^{\prime}$ as well as other functions of radius except $k_{\|}$stay constant in the absorption region one obtains (see also Ref. 24)

$$
P_{e} \approx \frac{S \omega \omega_{p e}^{2}\left|\tilde{E}_{\|}^{\prime}\right|^{2}}{\sqrt{32 \pi} v_{T e}\left|k_{\|}^{\prime}\right| \omega_{E}^{2}}\left(\omega-\omega_{e}+\frac{k_{\perp} T_{e}^{\prime}}{2 m_{e} \omega_{c e}}\right) \sim \frac{\delta_{e}}{\delta}\left(\frac{\nu_{e i}}{\omega_{E}}\right)^{1 / 2} P_{e, \mathrm{cold}},
$$

where the expression in the parentheses has been estimated as $\omega_{E}$. Finally, replacing in (19) $P_{e, \text { cold }}$ with smallest of $P_{e, \text { cold }}$ and $P_{e}$ given by (22), the relation between electron and ion torque is obtained as

$$
\frac{T_{(e) \varphi}^{(E M)}}{T_{(i) \varphi}^{(E M)}} \sim\left(\frac{\delta}{\delta_{V R}}\right)^{6} \min \left(1, \frac{\nu_{e i}}{v_{T e}\left|k_{\|}^{\prime}\right| \delta}\right)
$$

As one can see from Fig. 7 the torque acting on electrons appears to be very small compared to the torque acting on ions if one estimates the width of the resonance layer $\delta$ from the drift-MHD theory (Table I of Ref 12) using the the same anomalous viscosity coefficient as in Section II B, $\mu_{\perp}=10^{4} \mathrm{~cm}^{2} \mathrm{~s}^{-1}$. It should be noted, however, that drift-MHD values of $\delta$ appear to be roughly ten times smaller than ion Larmor radius, $\rho_{L i}=v_{T i} / \omega_{c i}$ (see 
Fig. 7), which invalidates the fluid approach. This feature of MHD theory in the case of finite ion temperature has been pointed out, in particular in Ref. 25. Computations with the kinetic model show that the actual layer width, in particular the radial scale of the largest component of the perturbed ion velocity $\tilde{V}_{i \perp}=\tilde{\mathbf{V}}_{i \perp} \cdot \mathbf{h} \times \nabla r$, does not shrink below $\rho_{L i} \approx 0.45 \mathrm{~cm}$ (see Fig. 8). The same radial scale is seen also for the parallel plasma response current density (see Fig. 13). Estimation of the torque ratio (23) with $\delta=\rho_{L i}$ shows that the electron torque is by an order of magnitude larger than the ion torque (see Fig. 7). Since also ion Landau damping and parallel ion viscosity contribute to the absorption of energy by ions, the values of ion and electron torque computed directly using the kinetic model are compared in Section III A.

\section{Effect of plasma response on the magnetic field configuration}

Results of field line tracing for the perturbation field in vacuum and in presence of plasma response currents are presented in Figs. 9 and 10 in real space and flux coordinates. In flux coordinates, also island widths are plotted which are obtained from the field line equations

$$
\begin{aligned}
\frac{\mathrm{d} \psi_{\text {tor }}}{\mathrm{d} \varphi} & =\frac{B^{\psi_{\text {tor }}}}{B^{\varphi}}=-\frac{\partial \tilde{A}_{\vartheta}}{\partial \varphi}\left(1+\frac{\partial \tilde{A}_{\vartheta}}{\partial \psi_{\text {tor }}}\right)^{-1}, \\
\frac{\mathrm{d} \vartheta}{\mathrm{d} \varphi} & =\frac{B^{\vartheta}}{B^{\varphi}}=\iota\left(1+\frac{\partial \tilde{A}_{\vartheta}}{\partial \psi_{\text {tor }}}\right)^{-1},
\end{aligned}
$$

where $\iota\left(\psi_{\text {tor }}\right)=1 / q\left(\psi_{\text {tor }}\right)=\mathrm{d} \psi_{\text {pol }}\left(\psi_{\text {tor }}\right) / \mathrm{d} \psi_{\text {tor }}$ is the rotational transform angle, for the case where only one harmonic is present in the perturbation vector potential (2) and, therefore, the field line equations (24) have an integral

$$
\psi_{\text {tor }}+\frac{m}{n} \psi_{\text {pol }}\left(\psi_{\text {tor }}\right)+2\left|A_{\vartheta ; m, n}\left(\psi_{\text {tor }}\right)\right| \cos \left(m \vartheta+n \varphi+\arg \left(A_{\vartheta ; m, n}\left(\psi_{\text {tor }}\right)\right)\right)=\text { Const }
$$

Invariant (25) follows immediately from the Hamiltonian form of Eqs. (24),

$$
\frac{\mathrm{d} \psi_{\mathrm{pol}}}{\mathrm{d} \vartheta}=-\frac{\partial \tilde{A}_{\vartheta}}{\partial \varphi}, \quad \frac{\mathrm{d} \varphi}{\mathrm{d} \vartheta}=q+\frac{\partial \tilde{A}_{\vartheta}}{\partial \psi_{\mathrm{pol}}} .
$$

Contours of this quantity give the flux surfaces including the island flux surfaces corresponding to $(m, n)$ resonance. Maximum width of island separatrix (island width) is computed from (25) numerically. In the particular case where $\left|A_{\vartheta ; m, n}\right|$ dependence on radius can be 
ignored, $\left|\mathrm{d} A_{\vartheta ; m, n} / \mathrm{d} \psi_{\text {tor }}\right| \ll 1$ and $\left|\mathrm{d}^{2} A_{\vartheta ; m, n} / \mathrm{d} \psi_{\text {tor }}^{2}\right| \ll\left|\mathrm{d} \iota / \mathrm{d} \psi_{\text {tor }}\right|$, one gets for the island width

$$
\Delta \psi_{\text {tor }} \approx 4\left|\iota\left(\psi_{\text {tor }}^{(\text {res })}\right) A_{\vartheta ; m, n}\left(\psi_{\text {tor }}^{(\text {res })}\right)\right|^{1 / 2}\left|\frac{\mathrm{d} \iota\left(\psi_{\text {tor }}^{(\text {res })}\right)}{\mathrm{d} \psi_{\text {tor }}^{(\text {res })}}\right|^{-1 / 2},
$$

where $\psi_{\text {tor }}^{(\text {res })}$ is the toroidal flux at the resonant surface, $\mathrm{d} \psi_{\text {pol }}\left(\psi_{\text {tor }}^{(\text {res })}\right) / \mathrm{d} \psi_{\text {tor }}^{(\text {res })}=\iota\left(\psi_{\text {tor }}^{(\text {res })}\right)=$ $-n / m$.

It can be seen from the figures that effect of the perturbation field on the magnetic field configuration in the core plasma is negligible small (island widths are reduced by a factor of 10 or more) for most of the modes except the $m=-7, n=3$ mode which is almost locked to the electron fluid.

In the zoom, Fig. 10, one can see that ergodicity appears starting from $s=0.97$ where an open ergodic field region is formed. This remaining ergodic layer is rather robust because screening of the perturbation field by plasma response currents decreases near the separatrix (see Section II B) and because of the stronger island overlapping caused by increase of the shear there.

\section{ESTIMATION OF QUASILINEAR AND NONLINEAR EFFECTS}

\section{A. Quasilinear evolution}

In the quasilinear approximation, the perturbation field modifies the equilibrium profiles of poloidal and toroidal plasma velocity as well as the profiles of plasma density and electron temperature. The way to estimate the change of plasma velocity and the effect of such a change on the penetration threshold is well known (see Refs. 12,18-20). In the long mean free path regime, the perturbation field interacts mainly with electrons (see Section II C) which results in a (mainly perpendicular) quasilinear force acting on electrons due to the poloidal and toroidal momentum transfer from the perturbation field. Such a force cannot be compensated by a small perpendicular electron viscosity and cause $\mathbf{F} \times \mathbf{B}$ radial drift of electrons which, in turn, leads to plasma polarization and modification of the equilibrium electric field. The Lorentz force due to this additional radial drift velocity balances the quasilinear force acting on electrons. The resulting acceleration of the $\mathbf{E} \times \mathbf{B}$ rotation looks like an acceleration of plasma ions directly by the quasilinear force. This acceleration continues until the change of the toroidal viscous force acting on ions modifies the radial ion 
flux so that it matches the electron flux and polarization stops. Finally, in a steady state, this additional ambipolar convective flux must be balanced by a change of the anomalous diffusion flux in expense of a reduced density gradient. Usually, only the effect of the quasilinear force on the rotation is considered ${ }^{12,18-20}$. However, the modification of plasma density and electron temperature can also be important at the very edge and in the case of mode locking because these modifications change the local electron (and ion) diamagnetic rotation velocity which is of the same importance for the screening effect as $\mathbf{E} \times \mathbf{B}$ plasma rotation. In order to estimate these effects, the toroidal momentum balance equation in the presence of quasilinear forces acting on electrons and ions from the perturbation field is re-derived in this section for general tokamak geometry in order to retain also quasilinear particle fluxes which are an intermediate result of this derivation. For simplicity momentum sources which determine the rotation velocity in absence of the perturbation field, in particular, the NBI momentum source, are omitted in this equation. These sources do not enter Eq. (37) which determines the rotation velocity change due to the perturbation field being of interest here. Flux surface average (average over the volume between neighboring flux surfaces) of the covariant toroidal component of the $\alpha$-species momentum equation can be cast to the following form (compare, e.g., to Ref. 26)

$$
m_{\alpha} n_{\alpha} \frac{\partial}{\partial t}\left\langle g_{\varphi \varphi} V_{(\alpha)}^{\varphi}\right\rangle=\frac{e_{\alpha}}{c} \sqrt{g} B^{\vartheta}\left(\left\langle\Gamma_{(\alpha)}^{r}\right\rangle-\Gamma_{(\alpha)}^{(N E O)}-\Gamma_{(\alpha)}^{(A)}\right)+\left\langle F_{(\alpha) \varphi}^{(V)}\right\rangle+\left\langle F_{(\alpha) \varphi}^{(E M)}\right\rangle
$$

where $m_{\alpha}, e_{\alpha}, n_{\alpha}, V_{(\alpha)}^{\varphi}$ and $\left\langle\Gamma_{(\alpha)}^{r}\right\rangle=\left\langle\boldsymbol{\Gamma}_{(\alpha)} \cdot \nabla r\right\rangle$ are $\alpha$-species mass, charge, density, contravariant component of the toroidal fluid velocity and flux surface averaged particle flux density, respectively, $\Gamma_{(\alpha)}^{(A)},\left\langle F_{(\alpha) \varphi}^{(V)}\right\rangle$ and $\left\langle F_{(\alpha) \varphi}^{(E M)}\right\rangle$ denote prescribed flux surface averaged anomalous particle flux density, co-variant toroidal components of the viscous force density and force density from the I-coil perturbation field (they have the dimension of torque density), respectively, and

$$
\Gamma_{(e)}^{(N E O)}=-\Gamma_{(i)}^{(N E O)} \equiv-\frac{c}{\sqrt{g} B^{\vartheta} e_{e}}\left\langle e_{e} n_{e} E_{\varphi}+R_{\varphi}^{(e i)}\right\rangle
$$

is the neoclassical particle flux density which is automatically ambipolar due to the charge neutrality, $e_{i} n_{i}+e_{e} n_{e}=0$, and $R_{\varphi}^{(i e)}=-R_{\varphi}^{(e i)}$. Here $R_{\varphi}^{(e i)}$ and $E_{\varphi}$ are the covariant components of the electron-ion friction force and of the inductive electric field, respectively. Toroidal co-variant component of the metric tensor, $g_{\varphi \varphi}=R^{2}$, and metric determinant $g$ entering (28) and (29) correspond to flux variables $(r, \vartheta, \varphi)$ which differ from variables of 
Section II by the re-defined flux surface label $r=r\left(\psi_{\text {tor }}\right)$ which has a dimension of radius and is fixed by the condition ${ }^{27}\langle|\nabla r|\rangle=1$. With this definition, the total particle flux is a product of averaged flux density $\left\langle\Gamma_{(\alpha)}^{r}\right\rangle$ and flux surface area

$$
S=\int_{0}^{2 \pi} \mathrm{d} \vartheta \int_{0}^{2 \pi} \mathrm{d} \varphi \sqrt{g}
$$

In order not to overload the notation subscript " 0 " is omitted in this section on the unperturbed quantities such as $n_{\alpha}, B^{i}, E_{i}$ etc.

For electrons, ignoring small inertia term (l.h.s. of (28)) electron flux density is readily obtained as

$$
\left\langle\Gamma_{(e)}^{r}\right\rangle=\Gamma_{(e)}^{(N E O)}+\Gamma_{(e)}^{(A)}-\frac{c}{\sqrt{g} B^{\vartheta} e_{e}}\left\langle F_{(e) \varphi}^{(E M)}\right\rangle .
$$

Time derivative of Gauss's law and continuity equations link particle fluxes with the unperturbed radial electric field by

$$
\left\langle g^{r r}\right\rangle \frac{\partial E_{r}}{\partial t}=-4 \pi\left(e_{i}\left\langle\Gamma_{(i)}^{r}\right\rangle+e_{e}\left\langle\Gamma_{(e)}^{r}\right\rangle\right)
$$

where $g^{r r}=|\nabla r|^{2}$ and it has been used that equilibrium electrostatic potential is close to a constant on the flux surface so that $E_{r}$ is also a constant there. Assuming that poloidal ion velocity is zero due to the damping of poloidal rotation by the parallel viscosity (gyrorelaxation effect),

$$
V_{(i)}^{\vartheta}=V_{i \|} \frac{B^{\vartheta}}{B}-\frac{c B_{\varphi}}{\sqrt{g} B^{2}}\left(E_{r}-\frac{1}{e_{i} n_{i}} \frac{\partial p_{i}}{\partial r}\right)=0
$$

where $p_{i}$ is ion pressure, the toroidal contra-variant velocity (toroidal angular frequency) is linked directly to the radial electric field and ion pressure gradient,

$$
V_{(i)}^{\varphi}=V_{i \|} \frac{B^{\varphi}}{B}+\frac{c B_{\vartheta}}{\sqrt{g} B^{2}}\left(E_{r}-\frac{1}{e_{i} n_{i}} \frac{\partial p_{i}}{\partial r}\right)=\frac{c}{\sqrt{g} B^{\vartheta}}\left(E_{r}-\frac{1}{e_{i} n_{i}} \frac{\partial p_{i}}{\partial r}\right) .
$$

Due to (B4) $V_{(i)}^{\varphi}$ is constant on the flux surface (plasma rotates within the flux surface as a solid body). Equation for time evolution of this quantity is obtained eliminating electric field in (32) with help of (34) and substituting there electron and ion flux densities using (31) and (28) for $\alpha=i$, respectively,

$$
\begin{aligned}
\left(1+\frac{v_{A}^{2}}{c^{2}} \frac{\left\langle g^{r r}\right\rangle g\left(B^{\vartheta}\right)^{2}}{\left\langle g_{\varphi \varphi}\right\rangle B^{2}}\right) m_{i} n_{i}\left\langle g_{\varphi \varphi}\right\rangle \frac{\partial V_{(i)}^{\varphi}}{\partial t} & =\left\langle F_{(i) \varphi}^{(V)}\right\rangle+\left\langle F_{(e) \varphi}^{(E M)}\right\rangle+\left\langle F_{(i) \varphi}^{(E M)}\right\rangle \\
& -\frac{1}{c} \sqrt{g} B^{\vartheta}\left(e_{i} \Gamma_{(i)}^{(A)}+e_{e} \Gamma_{(e)}^{(A)}\right)
\end{aligned}
$$


where $v_{A}=B / \sqrt{4 \pi n_{i} m_{i}}$ is the Alfven velocity. Assuming that in absence of the perturbation radial polarization current due anomalous non-ambipolar fluxes as well as other momentum sources which were omitted here for simplicity are balanced by the (anomalous) viscous force which has the form similar to the collisional viscous force,

$$
\left\langle F_{(i) \varphi}^{(V)}\right\rangle=\left\langle F_{(i) \varphi}^{(V)}\left(V_{(i)}^{\varphi}\right)\right\rangle=\frac{1}{S} \frac{\partial}{\partial r}\left(S m_{i} n_{i} \mu^{(\varphi)}\left\langle g_{\varphi \varphi}\right\rangle \frac{\partial V_{(i)}^{\varphi}}{\partial r}\right)
$$

such that toroidal viscosity coefficient (momentum diffusion coefficient) $\mu^{(\varphi)}$ is independent of $V_{(i)}^{\varphi}$, for the change in toroidal velocity due to the perturbation field, $\Delta V_{(i)}^{\varphi}$, one obtains the ordinary differential equation

$$
\left\langle F_{(i) \varphi}^{(V)}\left(\Delta V_{(i)}^{\varphi}\right)\right\rangle+\left\langle F_{(e) \varphi}^{(E M)}\right\rangle+\left\langle F_{(i) \varphi}^{(E M)}\right\rangle=0
$$

Using the boundary conditions

$$
\left.\frac{\partial \Delta V_{(i)}^{\varphi}}{\partial r}\right|_{r=0}=\left.\Delta V_{(i)}^{\varphi}\right|_{r=a}=0
$$

where $a$ is plasma radius, and the fact that quasilinear force in the case of a single perturbation mode is localized around $r=r_{\text {res }}$ in the narrow region $\left|r-r_{\text {res }}\right|<\Delta r$ integration of a steady state equation $(37)$ gives $^{12,19}$

$$
\Delta V_{(i)}^{\varphi}\left(r_{r e s}\right) \approx T_{\varphi}^{(E M)} \int_{r_{r e s}}^{a} \frac{\mathrm{d} r}{S m_{i} n_{i} \mu^{(\varphi)}\left\langle g_{\varphi \varphi}\right\rangle} .
$$

where

$$
T_{\varphi}^{(E M)}=T_{(e) \varphi}^{(E M)}+T_{(i) \varphi}^{(E M)}, \quad T_{(\alpha) \varphi}^{(E M)}=\int_{0}^{a} \mathrm{~d} r S\left\langle F_{(\alpha) \varphi}^{(E M)}\right\rangle=\int_{V} \mathrm{~d}^{3} r\left\langle F_{(\alpha) \varphi}^{(E M)}\right\rangle
$$

is the toroidal torque. Using $S \approx 4 \pi^{2} r R_{\text {axis }},\left\langle g_{\varphi \varphi}\right\rangle \approx R_{\text {axis }}^{2}$ one obtains an estimate

$$
\Delta V_{(i)}^{\varphi}\left(r_{r e s}\right) \sim \frac{T_{\varphi}^{(E M)}\left(a-r_{r e s}\right)}{4 \pi^{2} a R_{\mathrm{axis}}^{3} m_{i} n_{i}\left(r_{\mathrm{res}}\right) \mu^{(\varphi)}}
$$

The value of $T_{\varphi}^{(E M)}$ for a single mode can be estimated from Fig. 11 where the total toroidal torque and the torque on ions is shown for the mode $m=-7, n=3$ as a function of $V_{e \perp}=V_{e *}-V_{i *}+r V_{(i)}^{\varphi} q^{-1}$ where $V_{e *}$ and $V_{i *}$ are electron and ion diamagnetic velocities, respectively. This dependence has been obtained by scaling the original $V_{(i)}^{\varphi}$ shown in Fig. 1 by the factor changing between 0 and 2 . Here $V_{e \perp}$ and $V_{(i)}^{\varphi}$ are counted in directions of 
poloidal magnetic field and toroidal current, respectively. For low toroidal rotation velocities $V_{e \perp}<0$ due to $V_{e *}<0$ and, therefore, torque is positive, i.e. it leads to toroidal spin up. Estimating the torque away from the resonant peak as $T_{\varphi}^{(E M)} \sim 10^{5}$ dyne $\mathrm{cm}$ and $\mu^{(\varphi)} \sim 10^{4} \mathrm{~cm}^{2} \mathrm{~s}^{-1}$ one obtains for the spin up from a single mode $R_{\text {axis }} \Delta V_{(i)}^{\varphi} \sim 10^{5} \mathrm{~cm} \mathrm{~s}^{-1}$. Comparing Fig. 5 to Fig. 3 one can see that for all resonant modes except for $m=-5$ and $m=-6 V_{e \perp}<0$ and, therefore, all these modes lead to a toroidal spin up. Thus, total spin up is a few $\mathrm{km} \mathrm{s}^{-1}$. This estimate strongly depends, however, on the value of toroidal viscosity, $\mu^{(\varphi)}$, which is not a well determined quantity. It should be noted that the change in the rotation velocity is very small, about two orders of magnitude smaller that rotation velocity on the axis (see Fig. 1). Roughly this agrees with the value of the torque from the NBI which is about $8 \cdot 10^{7}$ dyne $\mathrm{cm}$.

It should be noted that due to the nonlinear dependence of the torque on $\Delta V_{(i)}^{\varphi}$ (see Fig. 11) Eq. (39) is nonlinear and has several roots which describe either the state where the islands "slip" through the plasma and perturbation is strongly screened by plasma response currents, or the "locked" state where the screening is strongly reduced ${ }^{12,19,20}$. In the reduced MHD theory one of the locked states is achieved if $V_{e \perp} \approx 0$. It can be seen from Fig. 5 and Fig. 11 that a locked state is observed only for the mode $m=-7, n=3$ (and other modes with the same helicity and different $n$ ). It is remarkable that the "resonant" value of $V_{e \perp}$ where the torque passes through zero and which is actually realized in the experiment is different from $V_{e \perp}=0$. This follows directly from kinetic theory ${ }^{11}$. Since the value of the torque in the vicinity of the "resonance" is more than an order of magnitude higher than elsewhere (which is the result of increased field amplitude due to better penetration near the "resonance"), locking of this mode prevents further spin up.

Besides the rotation change, modification of density profiles occurs if an essential amount of the torque is applied to electrons (see Eq. (31)). Actually torque on ions is two orders of magnitude smaller than torque on electrons (see Fig. 11). This modification is negligible if the additional flux due to the torque is small compared to the anomalous particle flux which for the estimates is assumed in the form $\Gamma_{(e)}^{(A)}=-D_{\perp} \partial n_{e} / \partial r$. Thus, the effect of the perturbation field on the density profile can be ignored if

$$
\frac{\Gamma_{(e)}^{(E M)}}{\Gamma_{(e)}^{(A)}}=\frac{c\left\langle F_{(e) \varphi}^{(E M)}\right\rangle}{\sqrt{g} B^{\vartheta} e_{e} D_{\perp}}\left(\frac{\partial n_{e}}{\partial r}\right)^{-1} \sim \frac{q T_{\varphi}^{(E M)}}{4 \pi^{2} r^{2} R_{\mathrm{axis}} \Delta r m_{i} \omega_{c i} D_{\perp}}\left(\frac{\partial n_{e}}{\partial r}\right)^{-1} \ll 1 .
$$


It is convenient to express the ratio (42) through the toroidal velocity change (39),

$$
\frac{\Gamma_{e}^{(E M)}}{\Gamma_{e}^{A}} \sim \frac{r_{r e s} \Delta V_{(i)}^{\varphi}}{v_{T i}} \frac{\rho_{L i}}{\Delta r} \frac{\mu^{(\varphi)}}{D_{\perp}} \frac{q R_{\text {axis }}^{2}}{r_{r e s}^{2}} \frac{r_{r e s}}{a-r_{r e s}}\left(\frac{r_{r e s}}{n_{i}} \frac{\partial n_{i}}{\partial r_{r e s}}\right)^{-1} \ll 1,
$$

where $v_{T i}=\sqrt{T_{i} / m_{i}}$ and $\rho_{L i}=v_{T i} / \omega_{c i}$ are ion thermal velocity and Larmor radius, respectively, and $\Delta r$ is the width of the resonance layer. It can be seen that for $\mu^{(\varphi)} \sim D_{\perp}$ condition (43) is usually well fulfilled in the core plasma.

It should be noted that the estimate of the local torque density $\left\langle F_{(e) \varphi}^{(E M)}\right\rangle \sim T_{\varphi}^{(E M)} /\left(4 \pi^{2} R_{a x i s} r_{\text {res }} \Delta r\right)$ used in (42) can be used if this quantity has predominantly a definite sign in the resonance layer. This is not the case for the locked mode $m=-7, n=3$ which can be seen from Fig. 12 where the radial profile of the local torque density computed directly by the Maxwell solver as

$$
\left\langle F_{(\alpha) \varphi}^{(E M)}\right\rangle=\left\langle\left(e_{\alpha} \tilde{n}_{\alpha} \tilde{\mathbf{E}}+\frac{1}{c} \tilde{\mathbf{j}}_{\alpha} \times \tilde{\mathbf{B}}\right) \cdot \frac{\partial \mathbf{r}}{\partial \varphi}\right\rangle
$$

is shown for the "slipping" mode $m=-5, n=3$ and for the pertinent locked mode. In fact, $\left\langle F_{(\alpha) \varphi}^{(E M)}\right\rangle$ is three orders of magnitude higher for the locked mode than for the slipping mode which is the consequence of better penetration and quadratic dependence of this quantity on the perturbation field amplitude. Estimating $D_{\perp}=10^{4} \mathrm{~cm}^{2} \mathrm{~s}^{-1}$ one obtains from the first equality in $(42)$ that $\Gamma_{(e)}^{(E M)} / \Gamma_{(e)}^{(A)} \sim 2>1$ for the locked mode. Thus, electron pressure profile must be affected by the perturbation field. As shown in Ref. 24,28, in the case of negligible finite Larmor radius effects which is well fulfilled for the electrons, expression for the particle flux $\Gamma_{(e)}^{(E M)}$ in the collisionless limit coincides with the expression for particle flux in the ergodic magnetic field ${ }^{29}$. Therefore, the expected consequence of perturbation induced transport is the elimination of electron pressure gradient in the resonance layer. If perturbation induced ion flux would be also large, ion pressure gradient would be eliminated too leading to complete locking of plasma rotation in the resonance layer. However, as one can see from Fig. 12, torque on ions is about 40 times smaller than torque on electrons and, therefore, it produces the radial ion flux which is smaller than anomalous. As a result, locking of $m=-7, n=3$ mode does not lead to zero rotation at $q=7 / 3$ rational surface.

\section{B. Nonlinear effects}

Nonlinear effects can be ignored if the island width is small compared to the width of the parallel current ${ }^{30}$. In this case, the re-distribution of the parallel current over the perturbed 
flux surfaces (including island flux surfaces) does not change significantly the radial profile of the parallel current density given by linear theory. This comparison is presented in Fig. 13. One can see that even for the "slipping" mode these widths are of the same order, and, strictly speaking linear theory is violated. However, this violation is weak, and therefore the results of the present analysis should be valid by order of magnitude. For the locked mode violation is much stronger. If the island opens nonlinearly, then $T_{m, n} \approx-2 m /\left(r \Delta^{\prime}\right)$ which is very close to unity for tearing stable modes ${ }^{18}$ (here $\Delta^{\prime}$ is a tearing mode stability index ${ }^{16}$ ). It should be noted that elimination of electron pressure gradient due to the quasilinear effects discussed in Section III A has not been modelled here. If the electron pressure gradient is absent in the resonance layer, the largest parallel electron response currents which are driven

by the Lorentz force from the perturbation field, $\tilde{F}_{L \|}=\left(e_{e} \mathbf{V}_{e} \times \tilde{\mathbf{B}} / c\right)_{\|}=-e_{e} V_{e \perp} \tilde{B}_{r} / c$ will be reduced to the level where perturbation magnetic field is described by the vacuum model which is close to the result of the nonlinear theory.

\section{EFFECT OF THE PERTURBATION FIELD ON THE PEDESTAL REGION}

As follows from the results of Section IID ergodization of the magnetic field is limited by plasma response currents to a relatively narrow region around separatrix, $s>0.97$, where, in turn, the perturbation field reaches almost the vacuum value. It is interesting to consider now the consequences of this result for plasma density and temperature in the pedestal region. Experimental density and temperature profiles in this region show the following features when the perturbation field is set on:

- Density gradient is almost unchanged by the perturbation field, profile shifts down as a whole. Changes of the shape can be seen only at the very edge $(s>0.97)$.

- Electron temperature is flat and ends with large gradient region at the last $2-3 \mathrm{~cm}$. With the perturbation it becomes even more flat, region of large gradient shrinks to about $1 \mathrm{~cm}$ (gradient increases).

- Ion temperature increases as a whole with the perturbation field.

- Change of profiles when varying the perturbation amplitude by factor of the order one is small. 
- Strong density and temperature changes occur in the long mean free path regime.

Most of these features can be explained by the change of boundary condition for the density at the pre-separatrix region which is affected by the ergodization. For this purpose, assuming that particle and electron heat transport are completely anomalous, the following model equations are considered,

$$
\begin{aligned}
\frac{\partial}{\partial r} S \Gamma_{e} & =0, & \Gamma_{e} & =-D_{\perp} \frac{\partial n_{e}}{\partial r} \\
\frac{\partial}{\partial r} S Q_{e} & =0, & Q_{e} & =-\frac{3}{2} D_{\perp}\left(T_{e} \frac{\partial n_{e}}{\partial r}+n_{e} \frac{\partial T_{e}}{\partial r}\right),
\end{aligned}
$$

where $\Gamma_{e}=\left\langle\Gamma_{(e)}^{r}\right\rangle$ and $Q_{e}$ particle and energy flux density, respectively. These equations follow, in particular, from Eqs.(4), (6), (61) and (62) of Ref. 31 for the electrostatic turbulence with small parallel wave numbers,

$$
\frac{\Delta k_{\perp}}{v_{T e} k_{\|}} \frac{\partial \omega}{\partial k_{\perp}} \gg 1
$$

where $\Delta k_{\perp}$ and $v_{T e}=\sqrt{T_{e} / m_{e}}$ are perpendicular spectral width and electron thermal velocity, respectively, in the case of small curvature drift effects. Thus, one can formally put $k_{\|}=\omega_{D \alpha}=0$ in Eqs.(61) of the pertinent reference. Ignoring for simplicity viscous tensor, collisional heat flux and equilibrium radial electric field, after the re-notation Eqs. (45) are obtained. If zero boundary condition is prescribed for the temperature at the separatrix, $T_{e}(a)=0$, Eqs. (45) link temperature and density profiles through a simple relation

$$
T_{e}(r)=\frac{2 Q_{e}}{3 \Gamma_{e}}\left(1-\frac{n_{e}(a)}{n_{e}(r)}\right),
$$

where $Q_{e} / \Gamma_{e}$ ratio is independent of the radius. According to Eqs. (45), the density profile does not change shape and moves as a whole if the boundary condition for the density at the separatrix, $n_{e}(a)$, changes. At the same time, electron temperature increases and steepens with the reduction of $n_{e}(a)$ (see Fig. 14). Increase and steepening of the temperature profile with density decrease is due to the reduction of the conductive heat flux fraction (linear in temperature gradient) as compared to the convective heat flux fraction (linear in temperature) which tends to form a constant temperature profile. As shown in Ref. 32, the increase of ion temperature is due to the fact that the anomalous diffusion coefficient is smaller than the neoclassical heat diffusion coefficient. Since neoclassical heat diffusion coefficient scales linearly with density, ion temperature increases due to the density decrease. 
Thus, the reasons for the density pump-out in the whole pedestal should be looked for in the narrow ergodized pre-separatrix region or outside the separatrix. This can be increased screening of neutrals due to the ergodization, or the effect of the perturbation field on the ion orbit losses. In the second case, without the perturbation field, orbit losses are minimized by the equilibrium radial electric field which develops due to relatively small mobility of electrons. Ergodization releases electrons and, therefore, changes the radial electric field which does not prevent ion orbit losses anymore. The mechanism connected with ion orbit losses should be more pronounced in the long mean free path regime where, in fact, major changes of the profiles are observed. It should have a property of "saturation" with increasing perturbation amplitude because as long as ergodization has completely destroyed the ambipolar electric field preventing the ion orbit losses, particle flux from this region becomes fully determined by the ion orbit loss mechanism which is independent of the ergodicity level.

\section{SUMMARY}

In this work, with the help of the linear kinetic model for the straight periodic inhomogeneous cylinder combined with 3D vacuum magnetic field data, the effect of plasma response currents on the non-axisymmetric magnetic field perturbations from the I-coil used for ELM mitigation in DIII-D has been studied for the particular experiment, shot 126006. It has been shown that a large perpendicular equilibrium electron fluid velocity leads to a significant (by two and more orders of magnitude) reduction of the perturbation field in the core plasma due to the screening of this field near the resonant magnetic surfaces. Both, the toroidal plasma rotation and the diamagnetic drift of electrons and ions contribute a similar amount to this velocity. In addition, the non-resonant plasma response leads to a relatively small (of the order one) attenuation of the perturbation field harmonics with negative poloidal wave numbers $m$ and to some amplification of harmonics with positive $m$ which are never resonant. It has been shown that in addition to the modification of the toroidal rotation velocity quasilinear effects lead also to modification of plasma density and electron temperature around resonant magnetic surfaces. However, all these effects appear to be small for the case considered here. In particular, the predicted change of the toroidal plasma rotation velocity by a few $\mathrm{km} / \mathrm{s}$ is two orders of magnitude smaller the velocity of 
plasma rotation due to the NBI injection. An estimation of non-linear effects shows that these effects approach the order of one and, therefore, the results of the present linear analysis are marginally valid. It has been observed that the kinetic model stays in approximate agreement with the reduced MHD model except in regions with fast perpendicular electron rotation. This is a remarkable fact because for plasma parameters used here, MHD theory predicts that the resonant layer width is more than by an order of magnitude smaller than ion Larmor radius and, therefore, MHD theory is formally invalid.

In contrast to the vacuum magnetic field model which predicts full destruction of the magnetic field configuration in the outer half of plasma volume it has been observed that plasma response currents significantly reduce the effect of the perturbation field on the magnetic configuration. In particular, the effect of the perturbation field on the magnetic surfaces in the core plasma is almost absent and an ergodic layer is formed only at the edge, for $s=\psi_{\text {tor }} / \psi_{\text {tor }}^{(a)}>0.97$, where $\psi_{\text {tor }}$ is the toroidal flux. The model predicts a toroidal plasma spin up (spin up of the ion component) which, in turn, leads to a simultaneous slowing down of the electron component and ends up with mode locking at the $q=7 / 3$ rational surface. A simple balance model for the pedestal region which assumes that the ergodization effect in the narrow pre-separatrix layer $s>0.97$ leads to a reduction of the plasma density in this layer, qualitatively reproduces the experimental behavior of plasma parameters in the pedestal region with the onset of the perturbation coil current. A possible reason for such a density reduction is the combined effect of ion orbit losses and magnetic field ergodization. The present results should be considered as a preliminary estimate for the following reasons. Although the linear approximation used here is shown to be marginally valid, poloidal mode coupling effects have been completely ignored in the present model. Near the separatrix these effects are of order one because the metric tensor of flux variables is essentially dependent on the poloidal angle there. Therefore, the results for the pedestal region should be viewed as an order of magnitude estimate only. In addition, the poloidal rotation velocity has been set to zero in this modelling. This approximation is good for the core plasma, but at the edge it can overestimate the perpendicular electron fluid velocity roughly by factor of 2 which also leads to a certain overestimation of the screening effect. However, this overestimation is not expected to change the order of magnitude of the results for the perturbation field amplitude. Thus this study can be viewed as a first estimate of important effects, while for the more reliable results a proper two dimensional modelling of the perturbation magnetic 
field is required.

\section{APPENDIX A: DIVERGENCE-FREE REPRESENTATION OF EQUILIBRIUM} AND VACUUM PERTURBATION MAGNETIC FIELD IN CYLINDRICAL COORDINATES

The unperturbed magnetic field is given in the divergence free form,

$$
\mathbf{B}_{0}=\nabla A_{0 \varphi} \times \nabla \varphi+B_{0 \varphi} \nabla \varphi
$$

where $B_{0 \varphi}$ is the co-variant toroidal magnetic field component (constant in the low pressure approximation) and $A_{0 \varphi}=-\psi_{\text {pol }}$ is the co-variant toroidal component of the vector potential. Here $\psi_{\text {pol }}=\psi_{\text {pol }}(R, Z)$ is the poloidal magnetic flux divided by $2 \pi$ which is provided by the equilibrium code $\operatorname{EFIT}^{13,14}$ on the $2 \mathrm{D}$ rectangular grid in cylindrical coordinates $(R, \varphi, Z)$ and is reconstructed by bi-cubic spline interpolation.

Perturbation magnetic field in vacuum is provided by the TRIP3D code $^{1}$ in the form of physical components, $\delta \hat{B}_{i}$, on an equidistant $3 \mathrm{D}$ grid in cylindrical coordinates. If these components are reconstructed by direct interpolation, $\nabla \cdot \delta \mathbf{B}=0$ condition is satisfied only up to the interpolation accuracy which may lead to appearance of attractors and numerical diffusion in the Poincaré mapping. For the divergence-free representation which satisfies this condition up to computer accuracy magnetic field is represented via vector potentials. Separating the axisymmetric part of the perturbation field, $\delta \overline{\mathbf{B}}$, as follows,

$$
\delta B^{i}(R, \varphi, Z)=\delta \tilde{B}^{i}(R, \varphi, Z)+\delta \bar{B}^{i}(R, Z)
$$

where components $\delta \tilde{B}^{i}(R, \varphi, Z)$ have zero average over $\varphi$, this part is presented as

$$
\delta \overline{\mathbf{B}}=\nabla \times\left(\bar{A}_{\varphi}(R, Z) \nabla \varphi\right)+\delta \bar{B}_{\varphi} \nabla \varphi
$$

where $\delta \bar{B}_{\varphi}=$ Const and

$$
\bar{A}_{\varphi}(R, Z)=\int_{R_{\min }}^{R} \mathrm{~d} R^{\prime} R^{\prime} \delta \bar{B}^{Z}\left(R^{\prime}, Z\right) .
$$

Here, $\delta \bar{B}^{Z}\left(R^{\prime}, Z\right)$ is the $Z$-component of the field averaged over the toroidal angle and $R_{\text {min }}$ is the inner boundary of the data box. The non-axisymmetric part of the field is represented 
using the gauge $\tilde{A}_{\varphi}=0$ as follows,

$$
\delta \tilde{\mathbf{B}}=\nabla \times \sum_{n=-\infty}^{\infty} \mathrm{e}^{i n \varphi}\left(A_{R, n}(R, Z) \nabla R+A_{Z, n}(R, Z) \nabla Z\right)
$$

where axisymmetric components of vector-potential $\tilde{\mathbf{A}}$ are zero, $A_{R, 0}=A_{Z, 0}=0$. The remaining Fourier amplitudes are obtained from Fourier amplitudes of the physical components of the magnetic field,

$$
A_{R, n}(R, Z)=\frac{i}{n} R \delta \hat{B}_{Z, n}(R, Z), \quad A_{Z, n}(R, Z)=\frac{-i}{n} R \delta \hat{B}_{R, n}(R, Z) .
$$

Since functions expanded in Fourier series are real, $f_{n}=f_{n}^{*}$, only half of the series is used in $(\mathrm{A} 5)$,

$$
f(\varphi)=\sum_{n=-\infty}^{\infty} f_{n} \mathrm{e}^{i n \varphi}=2 \operatorname{Re} \sum_{n=1}^{\infty} f_{n} \mathrm{e}^{i n \varphi}
$$

Fourier amplitudes of the physical magnetic field components $\delta \hat{B}_{R, n}, \delta \hat{B}_{Z, n}$ as well as averaged components $\delta \bar{B}^{Z}=\delta \hat{B}_{Z, 0}$ and $\delta \bar{B}_{\varphi}=R \delta \hat{B}_{\varphi, 0}(R, Z)$, respectively, are obtained using the approximate formulas for the Fourier transform of a periodic function $f(\varphi)$ given on an equidistant grid $\left(\varphi_{0}, \varphi_{1}, \ldots, \varphi_{K}\right)$ where $\varphi_{K}=2 \pi k / K$ as follows

$$
f_{n}=\frac{1}{K} \sum_{k=1}^{K} f\left(\frac{2 \pi k}{K}\right) \exp \left(-\frac{2 \pi i k n}{K}\right) .
$$

\section{APPENDIX B: CONVERSION TO FLUX COORDINATES BY INTEGRATION ALONG THE FIELD LINES}

Flux coordinates $\left(\psi_{\text {tor }}, \vartheta, \varphi\right)$ are generated by integration of the unperturbed field line equations in cylindrical variables,

$$
\frac{\mathrm{d} R}{\mathrm{~d} \varphi}=\frac{B_{0}^{R}}{B_{0}^{\varphi}}, \quad \frac{\mathrm{d} Z}{\mathrm{~d} \varphi}=\frac{B_{0}^{Z}}{B_{0}^{\varphi}}
$$

Denoting the solution which corresponds to the field line starting at the point $\left(R_{b}, 0, Z_{b}\right)$ with $R_{u}\left(R_{b}, Z_{b} ; \varphi\right)$ and $Z_{u}\left(R_{b}, Z_{b} ; \varphi\right)$, choosing $Z_{b}=Z_{\text {axis }}$ being the coordinate of the magnetic axis, and generating a set of these solutions for the grid of $R_{b}$ values (so that $R_{b}$ serves as a flux surface label), one obtains for the toroidal flux $\Psi_{\text {tor }}=2 \pi \psi_{\text {tor }}=\Psi_{\text {tor }}\left(R_{b}\right)$

$$
\begin{aligned}
\Psi_{\text {tor }}\left(R_{b}\right) & =\int_{0}^{2 \pi q} \mathrm{~d} \varphi B_{0}^{R}\left(R_{u}\left(R_{b}, Z_{\text {axis }} ; \varphi\right), Z_{u}\left(R_{b}, Z_{\text {axis }} ; \varphi\right)\right) \\
& \times R_{u}\left(R_{b}, Z_{\text {axis }} ; \varphi\right) Z_{u}\left(R_{b}, Z_{\text {axis }} ; \varphi\right)
\end{aligned}
$$


where the safety factor $q=q\left(R_{b}\right)$ is expressed through the period $\Delta \varphi$ of functions $R_{u}$ and $Z_{u}$ over the field line parameter $\varphi$ as follows, $q=\Delta \varphi /(2 \pi)$. Since in flux variables poloidal and toroidal angles satisfy on the field line the condition $q \vartheta-\varphi=$ Const, relations between cylindrical coordinates $(R, Z)$ and flux coordinates $\left(\psi_{\text {tor }}, \vartheta\right)$ are given by

$$
R=R_{u}\left(R_{b}\left(\psi_{\text {tor }}\right), Z_{\text {axis }} ; q\left(\psi_{\text {tor }}\right) \vartheta\right), \quad Z=Z_{u}\left(R_{b}\left(\psi_{\text {tor }}\right), Z_{\text {axis }} ; q\left(\psi_{\text {tor }}\right) \vartheta\right)
$$

Since the metric determinant of flux variables satisfies

$$
\sqrt{g} B^{\varphi}=q \sqrt{g} B^{\vartheta}=1
$$

the equilibrium magnetic field (A1) can also be presented as

$$
\mathbf{B}_{0}=\nabla \varphi \times \nabla \psi_{\text {pol }}+\nabla \psi_{\text {tor }} \times \nabla \vartheta
$$

According to Eqs. (A1) and (A2), axisymmetric part of the perturbation field provides small corrections to $A_{0 \varphi}$ and $B_{0 \varphi}$. Non-axisymmetric part of $\delta \mathbf{B}$ takes in flux variables the following form

$$
\delta \tilde{\mathbf{B}}=\nabla \tilde{A}_{\psi_{\text {tor }}} \times \nabla \psi_{\text {tor }}+\nabla \tilde{A}_{\vartheta} \times \nabla \vartheta
$$

Component $\tilde{A}_{\psi_{\text {tor }}}$ does not modify the magnetic field topology because this small term can be iteratively absorbed into the flux function. (Formally, $\tilde{A}_{\vartheta}$ can also be included in $\psi_{\text {tor }}$ however this would destroy the property of $\psi_{\text {pol }}$ to be a function of $\psi_{\text {tor }}$ alone and, therefore, destroy simple topology of the unperturbed magnetic field ${ }^{15}$.) Thus, both, the symmetric part of the perturbation field and vector potential component $\tilde{A}_{\psi_{\text {tor }}}$ can be ignored for small perturbation fields. Comparison of Poincaré plots computed for the vacuum perturbation field model with and without these correction terms show a negligible difference in the results. Therefore, these terms have been omitted in the further analysis.

Fourier amplitudes of the perturbed vector potential component $\tilde{A}_{\vartheta}$ are obtained during the field line integration as follows,

$$
A_{\vartheta ; m, n}\left(\psi_{\text {tor }}\right)=\frac{1}{2 \pi q} \int_{0}^{2 \pi q} \mathrm{~d} \varphi A_{\vartheta ; n}\left(R\left(R_{b}, Z_{\text {axis }} ; \varphi\right), Z\left(R_{b}, Z_{\text {axis }} ; \varphi\right)\right) \mathrm{e}^{-i m \varphi / q} .
$$

where

$$
A_{\vartheta ; n}(R, Z)=A_{R, n} \frac{\partial R}{\partial \vartheta}+A_{Z, n} \frac{\partial Z}{\partial \vartheta}=\frac{q}{B_{0}^{\varphi}}\left(B_{0}^{R} A_{R, n}+B_{0}^{Z} A_{Z, n}\right)
$$

and cylindrical components of vector potential are given by (A6). 
Field line tracing for the complete field $\mathbf{B}=\mathbf{B}_{0}+\delta \mathbf{B}$ is finally performed in cylindrical variables respectively transforming the amplitudes $A_{\vartheta ; n}$ modified by plasma response back to cylindrical variables.

\section{ACKNOWLEDGMENTS}

This work, supported by the European Communities under the contract of Association between EURATOM and the Austrian Academy of Sciences, was carried out within the framework of the Europe an Fusion Development Agreement. The views and opinions expressed herein do not necessarily reflect those of the European Commission. Additional funding is provided by the Austrian Science Foundation, FWF, under contract number P19889-N16, and by the US Department of Energy under DE-FC02-04ER54758 and DEFG02-04ER54698.

1 T. E. Evans, R. A. Moyer, and P. Monat, Phys. Plasmas 9, 4957 (2002).

2 T. E. Evans et al., Phys. Rev. Letters 92, 235003 (2004).

3 R. A. Moyer et al., Phys. Plasmas 12, 056119 (2005).

4 K. H. Burrell et al., Plasma Phys. Control. Fusion 47, B37 (2005).

5 T. E. Evans et al., Nature Phys. 2, 419 (2006).

6 T. E. Evans et al., Nuclear Fusion 48, 024002 (2008).

7 T. E. Evans et al., Journ. of Nuclear Materials 363-365, 570 (2007).

8 I. Joseph et al., Journ. of Nuclear Materials 363-365, 591 (2007).

9 I. Joseph et al., Calculation of the thermal footprint of resonant magnetic perturbations in DIII$D$ (Report at Biennial Workshop "Stochasticity in Fusion Plasmas",Juelich, Germany, March 05 - 07, 2007, Nuclear Fusion, submitted).

10 A. M. Runov et al., Phys. Plasmas 8, 916 (2001).

11 M. F. Heyn, I. B. Ivanov, S. V. Kasilov, and W. Kernbichler, Nuclear Fusion 46, S159 (2006).

12 A. Cole and R. Fitzpatrick, Phys. Plasmas 13, 032503 (2006).

13 L. L. Lao et al., Fusion Science and Technology 48, 968 (2005).

14 L. L. Lao et al., Nuclear Fusion 25, 1611 (1985). 
15 A. H. Boozer, Phys. Fluids 26, 1288 (1983).

16 H. P. Furth, P. H. Rutherford, and H. Selberg, Phys. Fluids 16, 1054 (1973).

17 R. Fitzpatrick and T. C. Hender, Phys. Fluids B 3, 644 (1991).

18 R. Fitzpatrick, Nuclear Fusion 33, 1049 (1993).

19 R. Fitzpatrick, Phys. Plasmas 5, 3325 (1998).

20 F. L. Waelbroeck, Phys. Plasmas 10, 4040 (2003).

21 Y. Kikuchi et al., Contrib. Plasma Phys. 46, 539 (2007).

22 M. B. Monagan et al., Maple 9 Advanced Programming Guide (Maplesoft, Waterloo, ON, Canada N2L 6C2, 2003), pp. $\mathrm{x}+444$.

23 J. B. Taylor, Phys. Rev. Letters 91, 115002 (2003).

24 I. B. Ivanov, S. V. Kasilov, W. Kernbichler, and M. Heyn, JETP Letters 86, 364 (2007).

25 B. Scott and F. Porcelli, Phys. Plasmas 11, 5468 (2004).

26 T. E. Stringer, Nuclear Fusion 35, 1008 (1995).

27 V. V. Nemov, S. V. Kasilov, W. Kernbichler, and M. F. Heyn, Phys. Plasmas 6, 4622 (1999).

28 M. F. Heyn, I. B. Ivanov, S. V. Kasilov, and W. Kernbichler, in 33rd EPS Conf. on Plasma Phys., Rome, 19-23 June 2006, ECA, edited by F. De Marco and G. Vlad (European Physical Society, Mulhouse, 2006), Vol. 30I, pp. P-4.174.

29 R. W. Harwey, M. G. McCoy, J. Y. Hsu, and A. A. Mirin, Phys. Rev. Letters 47, 102 (1981).

30 P. H. Rutherford, Phys. Fluids 16, 1903 (1973).

31 H. Sugama and W. Horton, Phys. Plasmas 2, 2989 (1995).

32 M. Z. Tokar et al., Phys. Rev. Letters 98, 095001 (2007). 


\section{FIGURES}

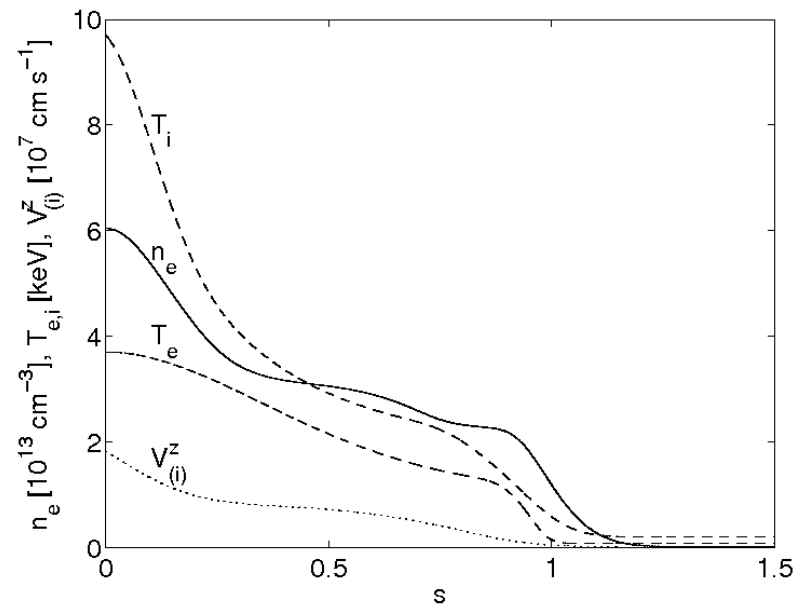

FIG. 1: Profiles of plasma density, temperature and toroidal velocity (counted in the direction of plasma current) vs the normalized toroidal flux $s=\psi_{\text {tor }} / \psi_{\text {tor }}^{(\text {a) }}$.

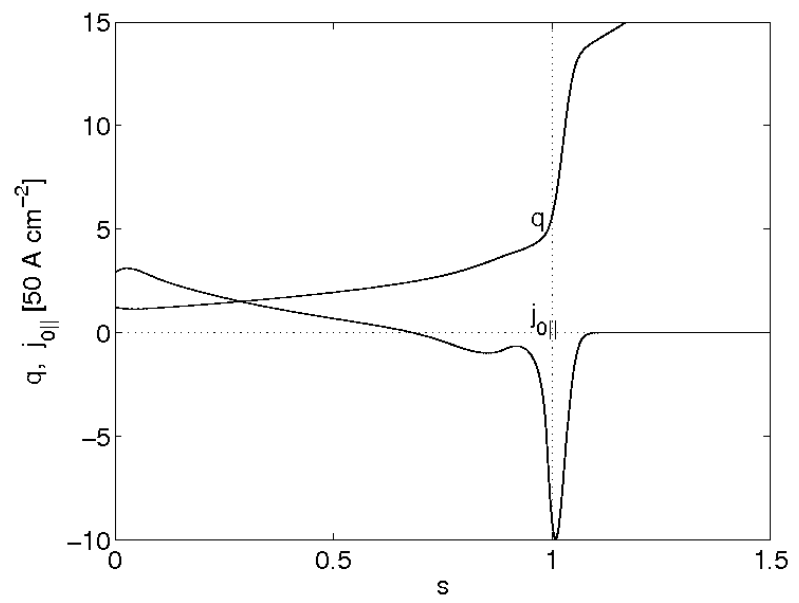

FIG. 2: Safety factor and parallel equilibrium current density vs the normalized toroidal flux $s$. 


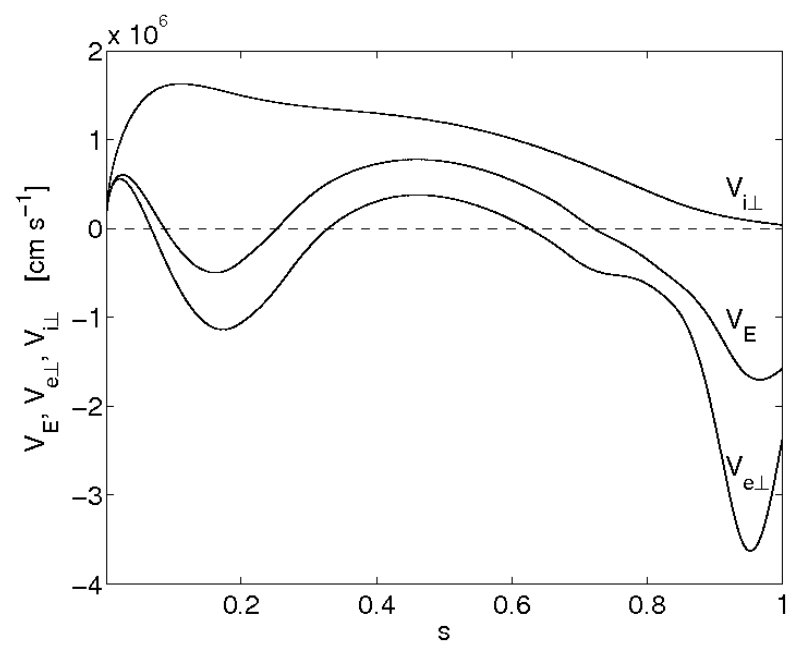

FIG. 3: Perpendicular equilibrium electron, $V_{e \perp}=V_{e *}+V_{E}$, and ion, $V_{i \perp}=V_{i *}+V_{E}$, fluid velocities and electric drift velocity $V_{E}$ vs the normalized toroidal flux $s$. Velocities are counted in the direction of poloidal magnetic field.

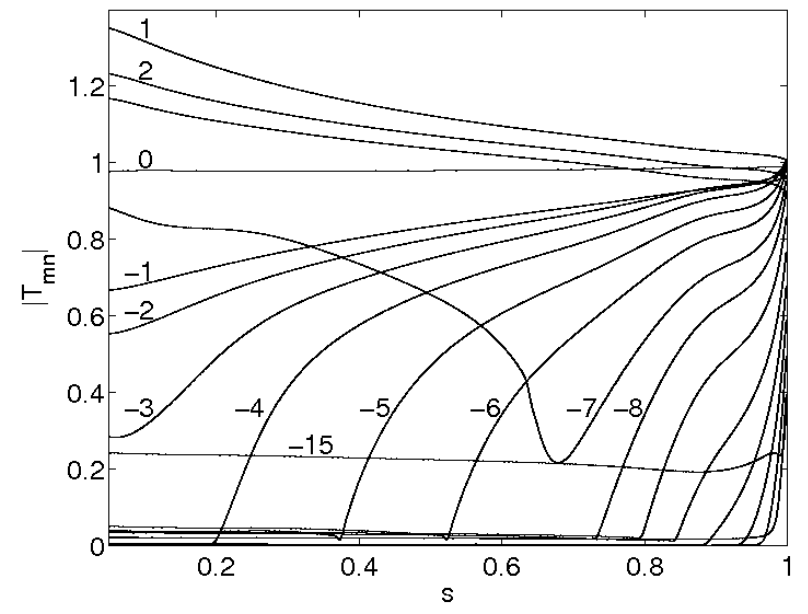

FIG. 4: Form factor modules $\left|T_{m, n}\right|$ vs the normalized toroidal flux $s$ for $n=3$. Poloidal wave numbers $m$ are given above the respective curves. 


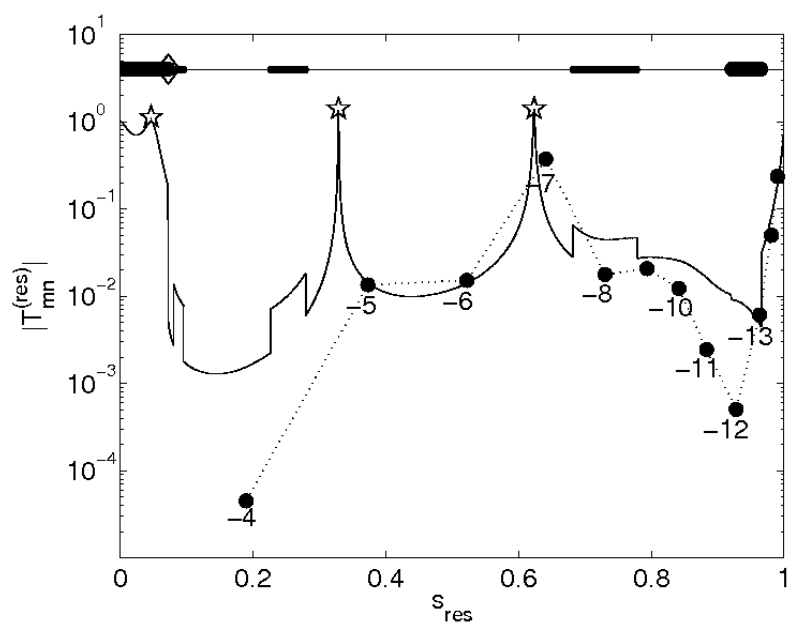

FIG. 5: Form factor modules at resonance magnetic surfaces as given by the kinetic model (black circles) and by the drift MHD theory (solid line) for $n=3$. Poloidal wave numbers of the resonant modes are indicated below the data points. Spikes marked by pentagrams on the drift MHD curve correspond to zeros of the perpendicular equilibrium electron fluid velocity $V_{e \perp}$ (see Fig. 3). The thickness of the horizontal line on top of the plot indicates the respective MHD regime of Ref. 12: thin - "first semicollisional", medium - "first Hall-resistive", thick - "inertial", diamonds - "visco-inertial".

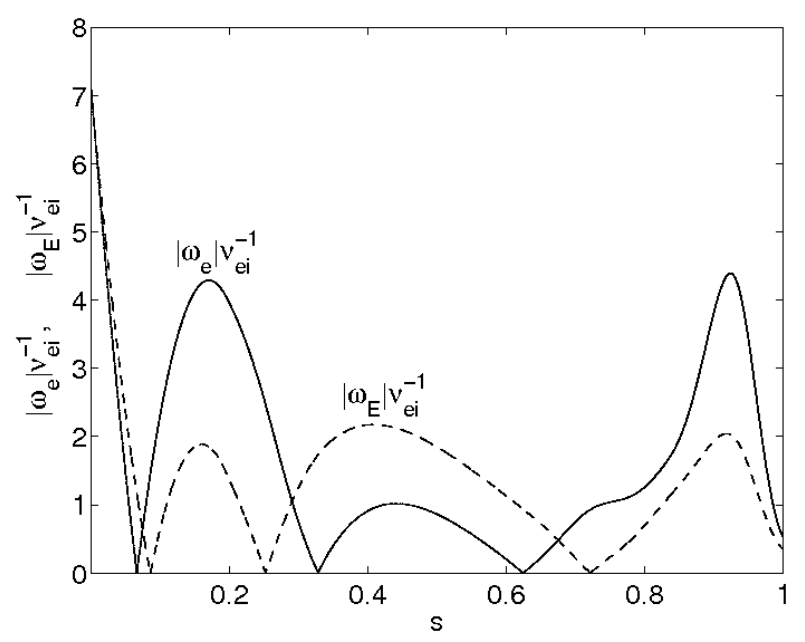

FIG. 6: Ratio of the $n=3$ perturbation frequency to the electron-ion collision frequency in the rest frame of electrons (solid) and in the reference frame where electric field is zero (dashed). As in Fig. 5, poloidal wave numbers are assumed to be continuous in order to match the resonance condition over the whole radius. 


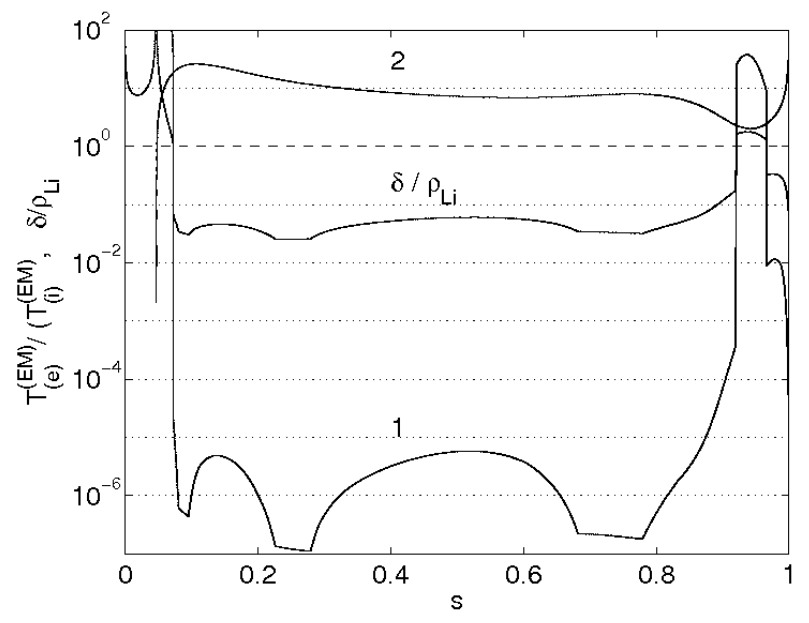

FIG. 7: Ratio of electron torque to ion torque estimated according to Eq. (23) for the layer width $\delta$ given by the MHD theory (curve 1) and putting $\delta=\rho_{L i}$ (curve 2). The ratio $\delta / \rho_{L i}$ is also shown.

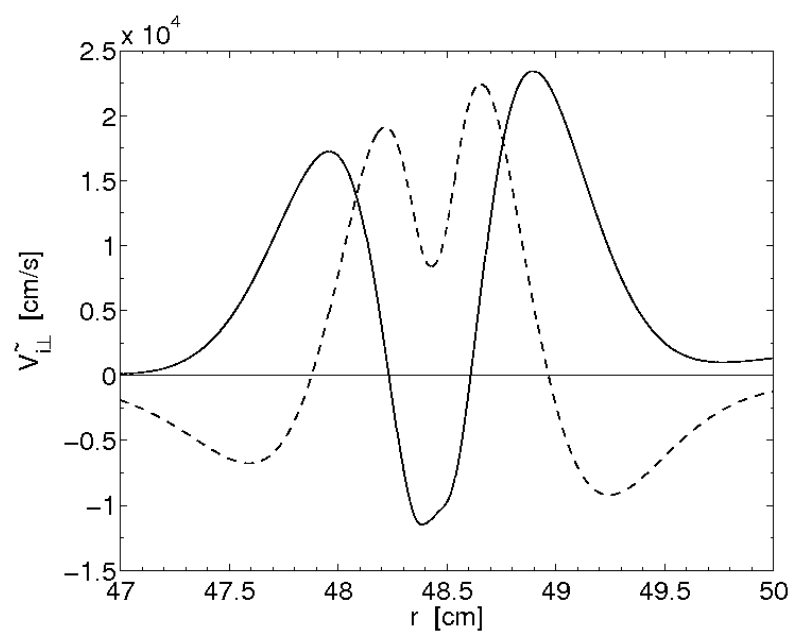

FIG. 8: Real (solid) and imaginary (dashed) parts of the perturbed ion velocity component $\tilde{V}_{i \perp}=\left(e_{i} n_{i 0}\right)^{-1} \tilde{\mathbf{j}}_{i} \cdot \mathbf{h} \times \nabla r$ for the mode $m=-5, n=3$ where $\tilde{\mathbf{j}}_{i}$ is the response current of ions (10). 

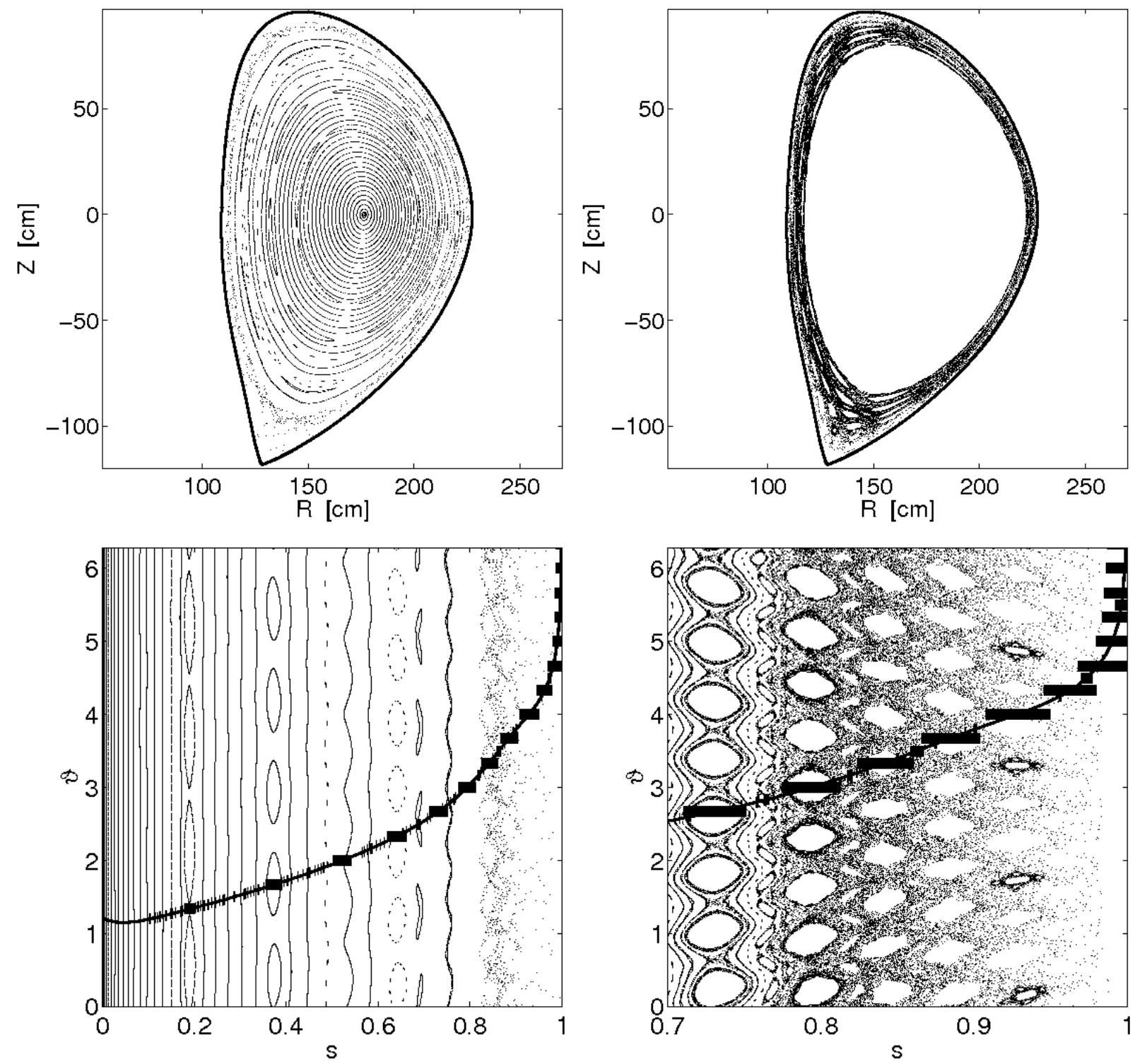

FIG. 9: Poincaré plots for the vacuum perturbation magnetic field model in real space (above) and magnetic (below) coordinates. Right figures present details of the region near the unperturbed separatrix with a higher density of the traced field lines. In magnetic coordinates, also the safety factor $q$ and analytical island widths corresponding to Eq. (25) are shown vs $s=\psi_{\text {tor }} / \psi_{\text {tor }}^{(\text {a) }}$. 

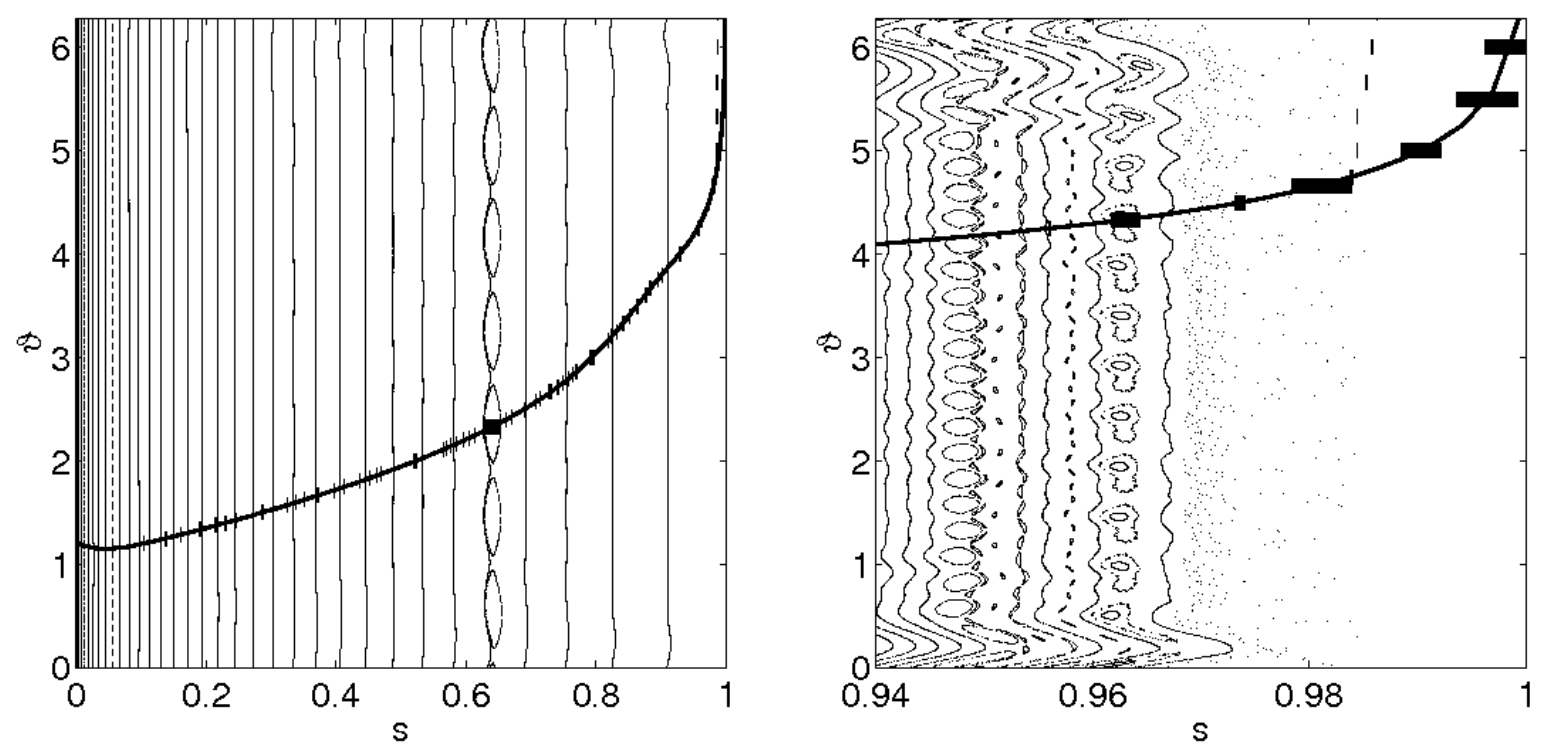

FIG. 10: Poincaré plot in magnetic coordinates for the perturbation magnetic field model with plasma response. See Fig. 9 for the notation. Right figure is a zoom of the region with the ergodic layer near the unperturbed separatrix.
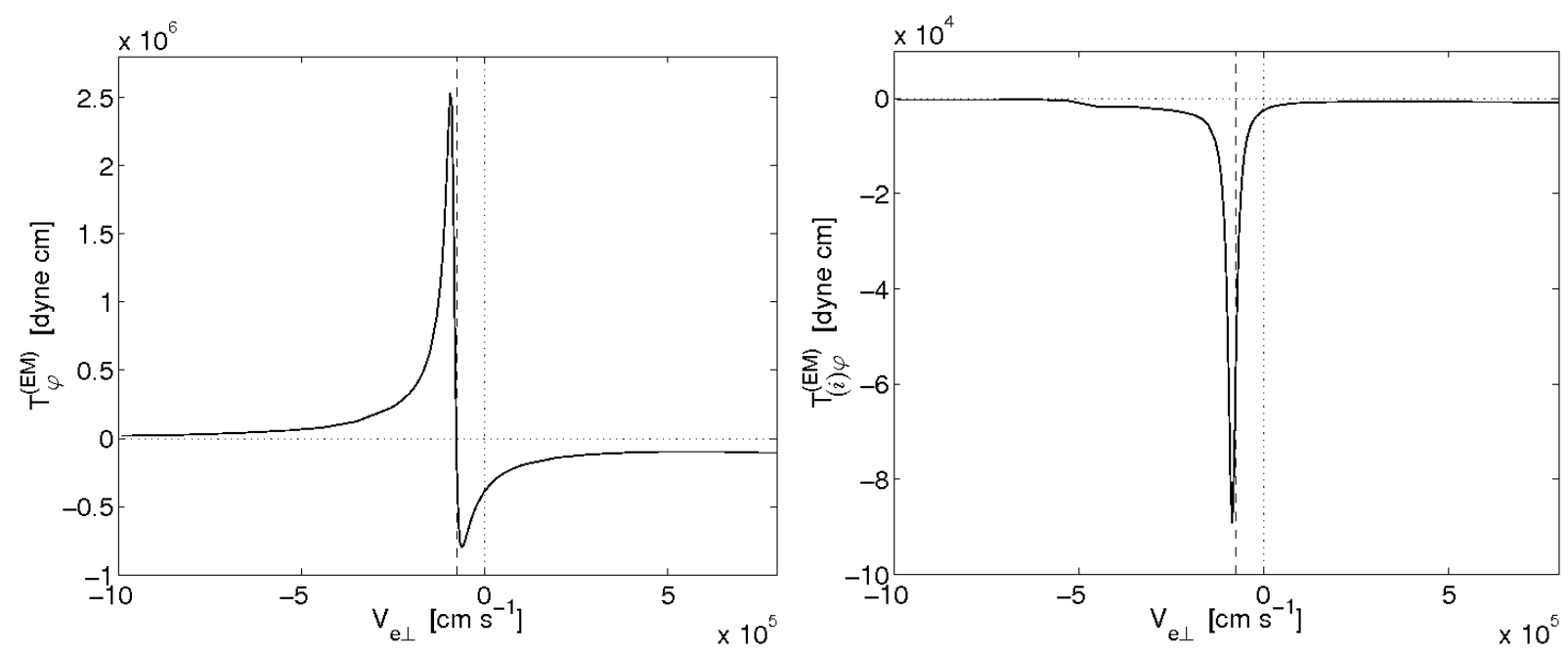

FIG. 11: Total toroidal torque (left) and torque on ions (right) for the mode $m=-7, n=3$ vs perpendicular equilibrium electron fluid velocity, $V_{e \perp}$. Torque is counted in the direction of plasma current. The variation of $V_{e \perp}$ from $-9.9 \cdot 10^{5} \mathrm{~cm} \mathrm{~s}^{-1}$ to $8.4 \cdot 10^{5} \mathrm{~cm} \mathrm{~s}^{-1}$ is obtained using as an input the toroidal velocity, $V_{(i)}^{z}$, equal to the experimental value times the scaling factor which changes from 0 to 2 . Dashed vertical line corresponds to the experimental value (scaling parameter 1 ). 

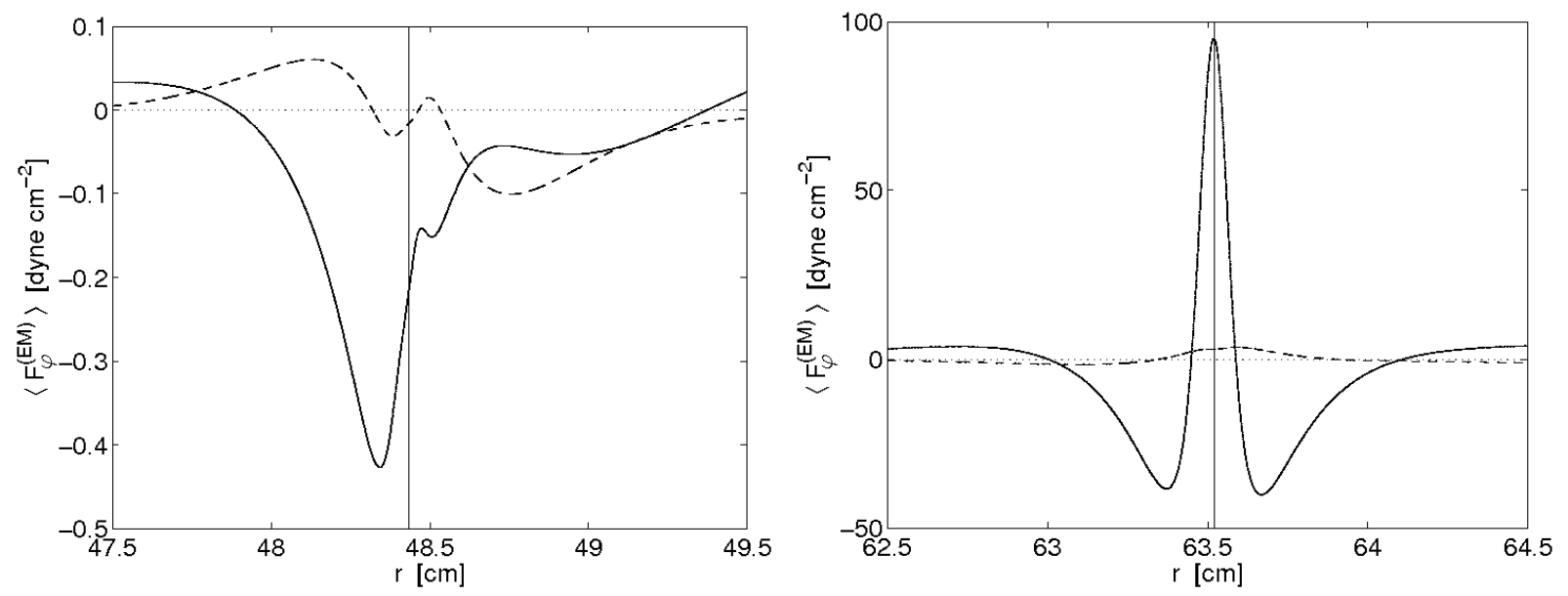

FIG. 12: Toroidal torque density, $\left\langle F_{\varphi}^{(E M)}\right\rangle$, for electrons (solid) and ions (dashed) for the "slipping" mode $m=-5, n=3$ (left) and for "locked" mode $m=-7, n=3$ (right). Vertical line indicates the position of resonant surface.
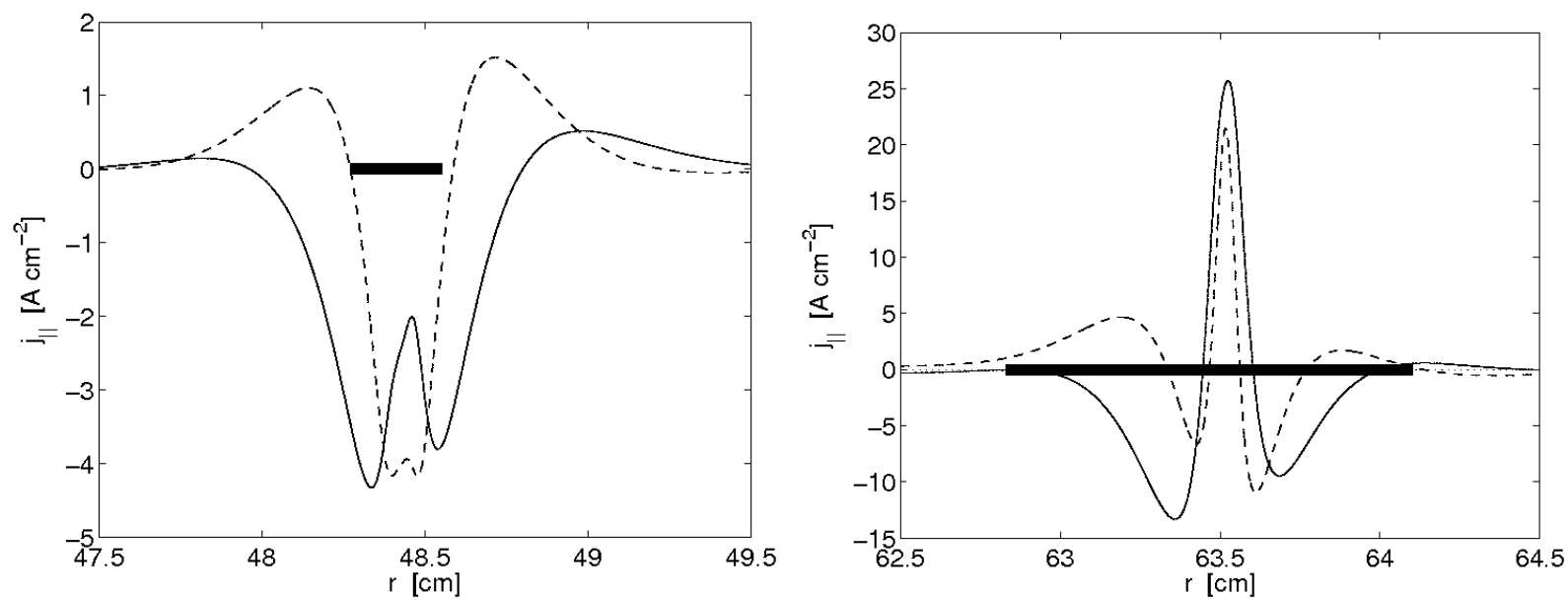

FIG. 13: Real (solid) and imaginary (dashed) parts of the Fourier amplitude of the parallel plasma response current, $j_{\|}$, for $m=-5, n=3$ (left) and for $m=-7, n=3$ (right) (in the vacuum, $A_{\vartheta ; m, n}$ is purely real). Thick horizontal lines indicate the positions of islands. 


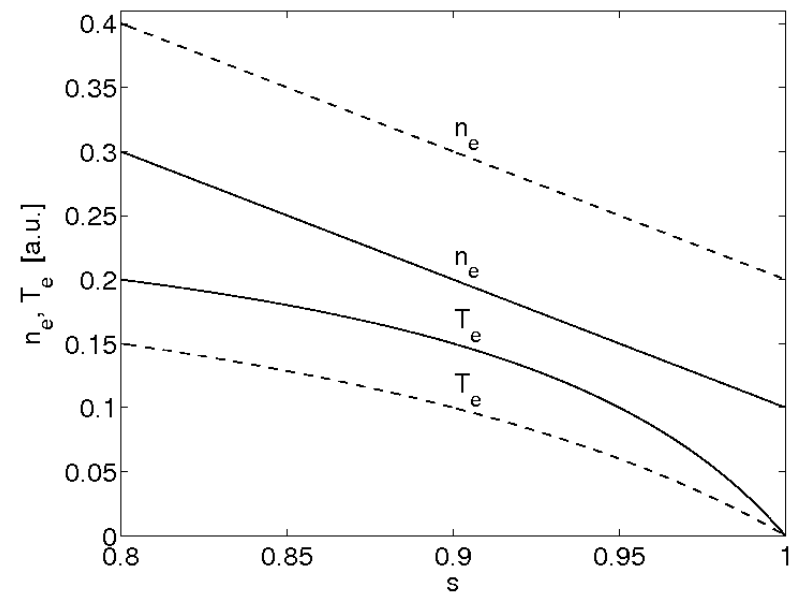

FIG. 14: Electron density and temperature resulting from Eqs. (45) before (dashed) and after (solid) reduction of the density at the separatrix. The same $D_{\perp}$ constant over the radius is assumed in both cases. 